\title{
Neuronal oscillations in the EEG under varying cognitive load: a comparative study between slow waves and faster oscillations
}

\author{
Charmaine Demanuele ${ }^{1,2}$, Samantha J. Broyd ${ }^{2}$, Edmund J. S. Sonuga-Barke ${ }^{2,3}$ and \\ Christopher James ${ }^{1,4}$ \\ ${ }^{1}$ Signal Processing and Control Group, Institute of Sound \& Vibration Research, University of \\ Southampton, UK \\ ${ }^{2}$ Institute for Disorders of Impulse \& Attention, Developmental Brain-Behaviour Laboratory, School \\ of Psychology, University of Southampton, UK \\ ${ }^{3}$ Department of Experimental Clinical and Health Psychology, Ghent University, Belgium \\ ${ }^{4}$ Institute of Digital Healthcare, WMG, University of Warwick, Coventry, UK
}

\section{Corresponding Author}

Charmaine Demanuele

Bernstein Center for Computational Neuroscience Heidelberg-Mannheim

Central Institute of Mental Health (ZI)

University of Heidelberg

Laborgebäude Room 2.10

J5 Mannheim, 68072

Deutschland

Email: c.demanuele@zi-mannheim.de

Tel: 004962117032367.

\section{Acknowledgements}

This work was supported by the Rayleigh Scholarship from the Institute of Sound and Vibration Research, and funding from the School of Psychology, University of Southampton. We acknowledge the support of our participants, Suzannah Helps (Developmental Brain-Behaviour Laboratory, School of Psychology, University of Southampton, UK) for her contributions to the task design, and Daniel Durstewitz (Central Institute of Mental Health, Mannheim, Germany) for useful comments on the manuscript. We also thank all three anonymous reviewers for their constructive comments.

\section{Keywords}

neuronal oscillations; slow waves; resting state EEG; event related potentials 


\begin{abstract}
Objective: This study has been specifically designed to investigate very low frequency neuronal oscillations (VLFO, $<0.5 \mathrm{~Hz}$ ) during resting states and during goal-directed tasks of graded difficulty levels, quantify the changes that the slow waves undergo in these conditions and compare them with those for higher frequency bands (namely delta, theta and alpha).

Methods: To this end we developed a multistage signal processing methodology comprising blind source separation coupled with a neural network based feature extraction and classification method.

Results: Changes in the amplitude and phase of brain sources estimates in the VLF band between rest and task were enhanced with increased task difficulty, but remained lower than those experienced in higher frequency bands. The slow wave variations were also significantly correlated with task performance measures, and hence with the level of task-directed attention.

Conclusions: These findings suggest that besides their prominent sensitivity to external stimulation, VLFOs also contribute to the cortical ongoing background activity which may not be specifically related to task-specific attention and performance.

Significance: Our work provides important insight into the association between VLF brain activity and conventional EEG frequency bands, and presents a novel framework for assessing neural activity during various mental conditions and psychiatric states.
\end{abstract}

\title{
Highlights
}

- We present a novel approach for exploring the functional relationship between very low frequency EEG oscillations (VLFO, $<0.5 \mathrm{~Hz}$ ) recorded at rest and those following the transition to goaldirected tasks of graded difficulty levels.

- We show that slow waves (i) are attenuated but not extinguished following a rest to task transition, and (ii) are sensitive to variations in cognitive load; (iii) the rest-to-task changes in the slow wave band correlate with performance measures and (iv) the changes are lower than those in higher frequency bands, tentatively suggesting the VLFOs' contribution to the cortical ongoing background activity.

- We present a new signal processing methodology for quantifying changes in band-limited EEG activity - this method, developed to gain insight into the neurophysiological role of the slow waves, could be explored with respect to altered functioning of brain oscillators in psychiatric and neurobehavioural disorders such as schizophrenia and ADHD. 


\section{Introduction}

The brain is believed to be a highly-distributed, self-organising system with different modules linked dynamically by synchronous oscillations (Varela et al., 2001; Buzsáki and Draguhn, 2004; Penttonen and Buzsáki, 2003). Transient assemblies comprising many neurons act in parallel in order to produce coherent perceptions, evaluations and behaviour depending on the task to resolve. These assemblies have a dynamic topography emerging as structures oscillating at multiple frequencies (delta $0.5-4 \mathrm{~Hz}$; theta 4-8 Hz; alpha 8-12 Hz; beta 13-20 Hz and gamma $25-100 \mathrm{~Hz}$ ), each associated with different aspects of cognition and brain states (Bear et al., 2001). A large body of literature has attempted to characterize the functional and clinical correlates of these relatively fast oscillations. Başar's empirically based theory of oscillatory neural assemblies (Başar, 1998:1999) suggests that these oscillations reflect the brain's information processing, each representing multiple functions selectively distributed in the brain; their activity and interactions are coordinated in cognition (Karakaş et al., 2000). Furthermore, these oscillators interact with each other and work together at various levels of synchrony; they can temporally co-exist in the same or different neural structures, and slower oscillators appear to group and modulate faster ones (Buzsáki and Draguhn, 2004; Balduzzi et al., 2008; He et al., 2008).

In addition, in recent years some studies have also demonstrated that neuronal oscillators go above and beyond the conventional EEG band $(0.5<f<50 \mathrm{~Hz})$ and potentially span frequencies from approximately $0.01 \mathrm{~Hz}$ to $600 \mathrm{~Hz}$ (Buzsáki and Draguhn, 2004). Particularly, very low frequency oscillations (VLFOs, $<0.5 \mathrm{~Hz}$ ) have been recorded in the EEG of preterm neonates, during epileptic seizure activity (Vanhatalo et al., 2005), in attention deficit hyperactivity disorder (ADHD) (Helps et al. 2008, 2009, 2010), and during sleep stages (Steriade et al., 1993; Vanhatalo et al., 2004). These VLFOs, first observed by Biswal et al. (1995) within the motor cortex in functional magnetic resonance imaging (fMRI) blood oxygenation level dependent (BOLD) signals during rest, appear to be intrinsically generated by the brain, are most prominent during rest and may allow temporal synchronisation to promote communication across diverse brain networks (Buzsáki and Draguhn, 2004; Fransson, 2005, 2006). It is proposed that these slow waves represent an intrinsic continuous process which is required to maintain self-awareness (Fransson, 2006), as well as awareness of our surroundings and of the passage of time (Fox and Raichle, 2007; Balduzzi et al., 2008). Moreover, brain imaging studies have shown that very slow fluctuations observed during the course of a task are smaller than those during rest (Fox and Raichle, 2005; Fox and Raichle, 2007; Sonuga-Barke and Castellanos, 2007). This suggests that they are, to some extent, being attenuated during task in order to allow goal-directed active processing to dominate. However, the so-called default-mode interference (DMI) hypothesis posits that this is not always the case - sometimes these oscillations may re-emerge during task, and if they did so, they compete and interfere with goal-directed processing and impair 
task performance by inducing periodic attentional lapses (Sonuga-Barke and Castellanos, 2007). Moreover, VLFOs are thought to contribute to inter-trial variability in evoked responses and behaviour (Fox et al., 2007; Fox and Raichle, 2007; Monto et al., 2008; Demanuele et al., 2010).

Despite the mounting empirical evidence and promising theoretical models (Laufs et al., 2003; Fox et al., 2006; Fox and Raichle, 2007; Leistner et al. 2007; Monto et al., 2008;), the mechanism of these slow rhythms remains as yet unclear, and more work is required to unravel the origin of this neural activity, its specific function, and the best way to assess and quantify it (Balduzzi, 2008, Rothenberger, 2009). To this end, we here present a focused study which has been specifically designed to explore the slow waves in the EEG under varying levels of attentional requirements. We set out to explore the functional relationship between the slow waves recorded during rest and those following the transition to goal-directed tasks of graded difficulty levels, and their plausible link with goal-directed attention. To gain perspective into the novel slow waves findings, we contrast them with those obtained for more conventional frequency bands, namely delta, theta and alpha oscillations.

Hence, throughout the study and in keeping with the literature previously discussed, the brain is conceptualised as a system comprising a number of band-limited sources or oscillators (Karakaş et al., 2000; Buzsáki and Draguhn, 2004). At rest, the system is in its most basic, spontaneous and "defaultmode" state. Stimulus-based tasks load the brain and tax the underlying oscillators eliciting a definitive standard evoked response in the EEG, namely the P300 (Polich, 2007). This event-related potential (ERP) has been associated with task-relevant attention and memory, and is highly sensitive to cognitive load (Karakaş et al., 2000; Herrmann and Knight, 2001; Datta et al., 2007; Polich, 2007; Smallwood et al., 2007). Its amplitude reflects the probability and task-relevance of a stimulus while its latency indicates the duration of stimulus evaluation (Polich, 2007). Consequently, these P300 parameters provide a useful index of the effect of varying the level of cognitive load on the brain.

In order to achieve this variable cognitive load, we implemented an experimental framework comprising two paradigms, namely, a sustained attention to response task (SART), also known as Go No-go paradigm (Datta et al., 2007), and a three-stimulus oddball (OB) task (Comerchero and Polich, 1999). The Go No-go task requires the participant to respond to one stimulus type (Go) and withhold a response to an infrequent stimulus (No-go) appearing in an unpredictable manner. As the name indicates, it assesses self-directed attention to the task at hand. The rhythmic nature of the task is designed to establish a relatively automatic, task-driven, prepotent response (Datta et al., 2007; Smallwood et al., 2007). This task has also been used to assess ERP markers of inhibition (e.g. Nogo N2; Falkenstein et al., 1999) and response preparation (Shibasaki et al., 1980). Meanwhile, the threestimulus oddball paradigm involves the recognition of an infrequent target stimulus from a sequence of more frequently occurring standard stimuli. The third stimulus is the infrequent non-target 
'distractor' that does not require a response but is there to disrupt the attentional system (Katayama and Polich, 1996; Comerchero and Polich, 1998; Hagen et al., 2006). This task is more complex than the SART, and engages a number of important resources including attention allocation, short term memory and response selection (Karakaş et al., 2000). Furthermore, the difficulty level of this paradigm can be adjusted by varying the perceptual discrimination difficulty between the target and standard stimuli (Comerchero and Polich, 1998; Hagen et al., 2006, Polich, 2007). In this study we utilized these three paradigms to create three task conditions, where the cognitive load would be varied in a graduated manner (higher loading implies greater task difficulty) from the easy and repetitive SART, to the more demanding OB Easy task, and finally to the most demanding OB Hard task. Each of the three task conditions were separated by a resting (no load) period.

Consistent with the study objectives, we propose the following data analysis procedure comprising:

(a) Preliminary analysis of the evoked potentials within task blocks.

ERP component measures (mainly the P300 amplitude and latency) are first used to assess the level of cognitive load placed on the brain, as well as to monitor the participants' performance during task execution (Herrmann and Knight, 2001). Behavioural data, such as reaction times, error rates and percentage of false alarms are also used to test this.

(b) Analysis and comparison of the EEG in default mode (i.e. during rest sessions) and during task execution.

Independent component analysis (ICA) is employed to isolate neurophysiologically meaningful components from the task and rest recordings separately. ICA (in its basic form) is a blind source separation (BSS) technique that extracts statistically independent and spatially distinct components (ICs) from a set of measured (mixed) signals, without using any additional information about the underlying (unknown) sources (James and Hesse, 2005; Ziehe and Müller, 1998; Demanuele et al., 2009). The extracted band-limited rest and task ICs are then filtered in four (slow, delta, theta and alpha) frequency bands, and their amplitude and phase features compared by means of a neural network methodology.

The new multistage signal processing methodology is made up of the following stages, as described in Section 2 and illustrated in Figure 1:

Stage (I). The application of BSS for denoising the data and extracting estimates of the underlying brain sources of interest (termed as independent components) during rest and task conditions.

Stage (II). Derivation of a set of features which describe the amplitude and phase of the independent components in different frequency bands.

Stage (III). Classification of band-limited features based on a neural network approach for pattern recognition, using the neuroscale algorithm and Gaussian mixture models (GMMs). 
Stage (IV). Further testing and analysis of: (a) classification results (derived from the GMMs' posterior probabilities); (b) test data; (c) correlation with behavioural data for assessing the link between rest-task variations of the band-limited brain activity and attention.

This analysis method provides us with a framework to objectively quantify the changes in bandlimited EEG activity under different levels of cognitive load.

\section{Materials and Methods}

\subsection{Participants}

The study was approved by the ethics committee of the School of Psychology at the University of Southampton in compliance with the University's ethics policy and the ethical standards laid down in the 1991 Declaration of Helsinki. Twenty-three healthy adult controls (age: $28.87 \pm 4.67 ; 11$ males, 12 females; post-graduate education level) were recruited and written informed consent was obtained. Before the EEG recording took place, the Barkley Adult ADHD rating scales and demographics data (re: epileptic seizures incidents, head injuries, vision or hearing problems, and the participants' use of medication or psychoactive substances) were collected for every participant. The former is an ADHD

self-reporting scale which contains 18 questions derived from the 18 ADHD symptom criteria for both inattention and hyperactivity/impulsivity factors reported in the DSM-IV. All participants scored in the normal range on this questionnaire. Moreover, none of the participants were taking any medication, none suffered from any head injuries or pathologies, and all had normal or corrected-tonormal vision.

\subsection{EEG Recordings}

The scalp direct current (DC) EEG was recorded from DC to $70 \mathrm{~Hz}$ using a Neuroscan SynAmps ${ }^{2} 70$ channel EEG system, sampling at $250 \mathrm{~Hz}$. Participants were fitted with an electrode cap (Easycap; Hersching, Germany) and EEG was recorded from 66 equidistant $\mathrm{Ag} / \mathrm{AgCl}$ electrodes, with a nose reference. The electrooculograph was recorded using electrodes above and below the left and right eye. Impedance for all electrodes was kept below $5 \mathrm{k} \Omega$. A high chloride, abrasive electrolyte gel was used to achieve a DC-stable skin-gel contact in order to ensure stable operation of the $\mathrm{Ag} / \mathrm{AgCl}$ electrodes (Vanhatalo et al., 2005).

\subsection{Experimental Paradigms}

\section{The Go No-go Task}

The Go No-go task consisted of two 10-minute task blocks separated by a 6-minute rest block. During this task single, randomised digits from 1 to 9 were presented on the computer screen at a regular, invariant rate. Participants were asked to use the index finger of their preferred hand to press a single button for each digit as it appears with the exception of the digit 3, as in the work by Datta et al. 
(2007). Stimuli were presented with a visual angle of $3.05^{\circ}$, every 1.2 seconds for a $250 \mathrm{~ms}$ duration, implying a total of 500 stimuli per task block, amongst which 60 (i.e. 12\%) were No-go stimuli. During the rest block participants were asked to look at the computer screen in a relaxed manner while focusing on a fixation cross at the centre of the screen in order to avoid extraneous movement.

\section{The Three-Stimulus Oddball Task}

Participants were asked to complete a 22-minute three-stimulus oddball visual task, consisting of two 8-minute task blocks separated by a 6-minute rest block. In this task, the target stimulus (probability of occurrence of 0.12 ) was a blue circle $4 \mathrm{~cm}$ in diameter (visual angle of $3.81^{\circ}$ ), and the distractor stimulus (probability of occurrence of 0.12 ) was a $16 \mathrm{~cm}^{2}$ square with a black and white checkerboard pattern $\left(1 \mathrm{~cm}\right.$ checks, visual angle of $\left.3.81^{\circ}\right)$. As for the target, the standard stimulus was a blue circle (probability of occurrence of 0.76 ), the diameter of which was systematically altered from $2.8 \mathrm{~cm}$ (visual angle of $2.67^{\circ}$ ) for the Easy to $3.6 \mathrm{~cm}$ (visual angle of $3.44^{\circ}$ ) for the Hard task conditions. This was done in order to grade the perceptual discrimination difficulty, and increase error rates and response time across task conditions, as in the work by Comerchero and Polich (1999). The stimuli were presented once every 1.2 seconds for a $250 \mathrm{~ms}$ duration, (i.e. 400 stimuli per task block). Stimuli presentations were randomised and condition order counterbalanced across participants. Participants were given a practice block consisting of 15 stimuli before each condition. As for the Go No-go task, during the rest block participants were asked to focus at the fixation cross at the centre of the screen.

The order of the two tasks was counterbalanced across participants. Throughout the recordings participants were seated on a comfortable chair in a quiet room free from distractions and with the LCD screen standing $60 \mathrm{~cm}$ away. They were asked to avoid unnecessary head movements and to respond to the task at hand 'as quickly and as accurately as possible'. After the first task participants were allowed to take a break until they felt ready to continue with the experiment. The length of the experiment recordings was specifically chosen to accommodate the long-period slow waves.

\subsection{Preliminary data analysis}

EEG data recorded at $250 \mathrm{~Hz}$ was low pass filtered offline at $16 \mathrm{~Hz}$, downsampled offline to a sampling frequency of $100 \mathrm{~Hz}$, and detrended to remove VLF DC shifts. Gross artifact laden channels, arising mainly from faulty electrodes in the electrode-cap, were replaced by the average of their neighbouring channels and the overall dataset was re-referenced to the average of all the channels. Twenty-five out of the 66 channels were then selected for the subsequent analysis, their position corresponding to the electrode locations of the International 10-20 System.

Every recording (i.e. one participant, one task block), was first decomposed by the temporal decorrelation source separation (TDSEP) ICA algorithm (Ziehe and Müller, 1998), artifactual 
components (such as ocular and muscle artifacts) were removed, and the resultant ICs were projected back onto the measurement space in order to investigate the coherent average of the P300 responses for various stimuli and task conditions. Analysis of performance measures, namely reaction times (RT), error rates and false alarms, was also carried out for both tasks. Reaction times were obtained from the logged button-press box responses. This was done in order to assess whether the required task environments (with variable difficulty levels) were successfully created by the task protocols, and to determine how well participants carried out each experiment.

\subsection{Comparison of brain activity during Rest and Task: A Multistage System}

The experimental procedure led to three task-environments: (a) Go No-go and Rest (simplest); (b) OB Easy and Rest; (c) OB Hard and Rest (hardest), as previously explained. Here we describe in detail the multistage signal processing methodology shown in Figure 1. For illustration purposes, we first demonstrate each stage on a subset of EEG recordings of ten participants during the OB Hard task environment (i.e. OB Hard - Rest).

Stage (I). The application of BSS for denoising the data and extracting estimates of the underlying brain sources of interest (termed as independent components) during rest and task conditions.

\section{(a) ICA procedure}

The 8-minute OB Hard recordings were divided into 4-minute segments ( $1^{\text {st }} 4$ minutes: $0-4$ minutes, $2^{\text {nd }} 4$ minutes: 4-8 minutes, and an overlapping segment: 2-6 minutes). For the Rest period, a 4-minute segment (1-5 minutes into the 6-minute rest block) was considered. This length was chosen to accommodate an adequate number of low frequency cycles, including infraslow $(<0.1 \mathrm{~Hz})$ oscillations. TDSEP algorithm - a specific ICA algorithm that achieves source separation by minimising temporal cross-correlations between the output signals - was employed on each segment (Ziehe and Müller, 1998). TDSEP was chosen since, unlike higher order statistics based methods such as FastICA (Hyvärinen, 1999), it exploits the strong temporal structure inherent in ERP signals. Figure 2 (a),(i) shows 5 of the 25 Task ICs of one participant during task execution; note the clear eye blink component (IC9), a low frequency component (IC11) and the strongest event related (ER) ICs (IC 2, 23 and 24) with central and parietal spatial distributions typical of the P300 complex. Meanwhile, the Rest ICs in Figure 2 (a),(ii) show most power in the frontal regions and in occipital regions (visual processing of fixation point, and alpha power associated with relaxation and absence of taskengagement).

(b) Separation of the task ICs into ER-ICs (related to task processing) and Background-ICs (reflecting ongoing brain activity)

Implementation of ICA on data recorded during task sessions renders three types of ICs: ER (taskrelated) ICs such as those associated with the P300-response, ICs constituting the background brain activity (Task-background ICs) and artifacts. This grouping is commonly done by visual inspection of 
the topographies and time series of the components. Here we apply the method proposed in Demanuele et al., (2009) where task ICs are hierarchically clustered based on their coherent average, as illustrated in Figure 2 (b). This procedure allows us to group together ICs with similar morphology in a more objective manner.

Meanwhile, the Rest ICs do not exhibit a specific response and should represent the activity of the brain in default mode, i.e. in the absence of stimulation. However, visual inspection of the Rest ICs after the removal of obvious artifactual components showed that three types of components were manifested in each recording, mainly: ICs with a slow and low-delta time-signature, those with an intermediate (high-delta and theta) frequency content, and those with a dominant alpha frequency content. Therefore, for better understanding of brain activity during the resting state, the Rest ICs were ordered based on their frequency content. For each IC, the ratio of power in the slow and low-delta frequency band $(0<f<1 \mathrm{~Hz})$ to the total power in $\left(0<f<f_{s} / 2 \mathrm{~Hz}\right)$ was found and the ICs were grouped accordingly, as shown in Supplementary Figure S1. Note that this grouping procedure is suboptimal and is only done to aid in the interpretation of results in later stages.

Stage (I) was repeated for each participant in order to form a databank of Task-ER, Task-Background (Bgd) and Rest ICs, (this essentially forms a training databank with 3 classes). Across the ten participants, TDSEP and hierarchical clustering procedures rendered a databank of 371 Task-ER and 316 Task-Background ICs after hierarchical clustering. A total of 230 ICs were extracted from the Rest period. Note that artifactual ICs, such as eye-blinks and eye movements, were immediately eliminated based on their morphology and topography.

Next, we needed to assess the differences between the three classes (Task-ER, Task-Background and Rest) as a function of frequency to gain insight into how the presence or absence of stimulation (i.e. external loading) was affecting the brain's oscillators.

Stage (II). Derivation of a set of features which describe the amplitude and phase of the ICs in different frequency bands.

(a) The databank ICs filtered in the frequency bands of interest

The ICs were filtered in four frequency bands: slow $(0<f \leq 0.5 \mathrm{~Hz})$, delta $(0.5<f \leq 4 \mathrm{~Hz})$, theta $(4<f \leq 8 \mathrm{~Hz})$ and alpha $(8<f \leq 12 \mathrm{~Hz})$ band, and for each band-limited IC the instantaneous amplitude and phase were obtained using the Hilbert transform (Le Van Quyen et al., 2001; Demanuele et al., 2009).

(b) A set of features extracted for each filtered IC

In order to characterise the changes in the instantaneous amplitude or phase of the ICs at one particular frequency band, these time series were individually parameterised with $p=12^{\text {th }}$ order auto-regressive (AR) models (Hayes, 1996). 
Stage (III). Classification of band-limited features based on a neural network approach for pattern recognition

(a) A distance measure between the AR model parameters

The AR models capture the structure of the amplitude and phase time series for each IC across the 4minute segment considered. The distance between ICs (i.e. the degree of similarity between the ICs belonging to one class and dissimilarity between ICs of different classes) was estimated by evaluating the Itakura-Saito Distance (ID) (Itakura, 1975) between two given sets of AR parameters for each pair of ICs amplitude or phase time series (Kong et al., 1995; Estrada et al., 2005).

An example of the symmetric ID matrix between the parameters of the AR models for the instantaneous amplitude of the ICs in the slow wave band during the Oddball Hard task environment is illustrated Figure 2 (c). The ID between the ICs features pertaining to the same class should be close to zero (blue); whilst that between different classes should be higher (tending towards red). Here, note the difference in the ID of the three classes, where ER class contains parameters corresponding to ICs 1:371, the Background (Bgd) contains parameters of ICs 372:687 whereas the parameters of ICs 688:917 form the Rest Class. It is already evident that there is overlap between the Rest class with both the Background and ER classes: the features of the Rest ICs with a slow morphology overlap with the Background class (hence the continuation from the Background into the Rest for 687:805), whilst the features of the Rest ICs with a higher frequency morphology (806:971) overlap with the ER class.

(b) A nonlinear transformation of this $P$-dimensional space $(p+1=P)$ onto a 2-D feature space

At this stage there is a $P$-dimensional space representing the ICs in the three classes; $P=13$ (set of features based on $12^{\text {th }}$ order AR models and ID method). The next step was to perform a nonlinear transformation of this $P$-dimensional space into a 2-D space for better visualisation of the natural divisions already apparent in the data. This was implemented through a neural network approach known as the supervised Neuroscale algorithm. Neuroscale is a clustering process which performs a dimension-reducing, nonlinear transformation of the original input data (Lowe, 1993; Lowe and Tipping, 1996; Nabney, 2004). The supervised version of this algorithm (Low and Tipping, 1996:1997) allows for the inclusion of subjective knowledge, regarding the expected relative differences between the classes, to be included in the training process. This is done by means of a distance matrix $s$ shown in Figure 2 (d), and a parameter $\alpha$ which varies from 0 (unsupervised) to 1 (completely supervised); see Appendix for more information.

Figure 2 (e) shows an example of the supervised neuroscale algorithm 2-D output (trained using the number of basis functions centres $k=80$ (chosen by trial and error) and $\alpha=0.5$ ), and based on the ID matrix of Figure 2 (c) and the $s$ matrix in Figure 2 (d). This result shows the ICs slow wave amplitude 
features occupy specific locations in the 2-D space, with the ER and Background classes being very well separated, whilst the Rest class overlaps with both task classes. This demonstrates that although the neuroscale clustering process can separate the three classes, both the features and the classifier itself are not perfect, which leads to misclassification of a number of input data points. Moreover, the classes themselves naturally possess overlapping traits. For this reason, the PDF space formed by the three classes must be characterised in order to be able track the probability of classification per class, i.e. the probability of each data point (in this 2-D space) belonging to each of the three classes. This was done via Gaussian mixture models (GMMs) (Bishop, 1995; Nabney, 2004).

(c) GMMs derived to track the probability of classification of the ICs in each of the three classes based on one particular set of features.

In this step, a set of GMMs were obtained in order to define the distributions of the neuroscale output (see Appendix for more information). Figure 2 (f) shows the contour plots for these GMMs. The posterior probabilities obtained by each GMM, namely $P_{\mathrm{A}}, P_{\mathrm{B}}$ and $P_{\text {mix }}$, were normalised such that together they added up to one, as illustrated in Figure 3.

\section{Stage (IV). Further testing and analysis}

Given the GMM posterior probabilities (Figure 3), the statistical error for the classification based on a particular set of features could then be derived by considering the maximum class posterior probability for each data point representing one IC. For example, for the first data point (representing $\left.\mathrm{IC}_{1}\right) P_{\mathrm{A}}$ was $0.82, P_{\mathrm{B}}$ was 0.08 and $P_{\text {mix }}$ was 0.1 , thus it was classified as belonging to process A, i.e. it was an ER task IC. The interpretation (for class A only) is given in Table 1 (Linn, 2004).

The sensitivity of classification for class A was then defined as

$$
\text { Sensitivity }_{\text {class A }}=1-\mathrm{FNR}_{\text {class A }} \text {, }
$$

where $\mathrm{FNR}_{\text {class } \mathrm{A}}$ is the false negative rate for class A given by

$$
\mathrm{FNR}_{\text {class } \mathrm{A}}=\frac{\text { number of ICs that belong to class A misclassified as class B or C }}{\text { total number of ICs that belong to class A }} .
$$

These indices for each class were portrayed in a confusion map. In the normalised map of Figure 4 the diagonals show the sensitivity of classification for each class. The off-diagonal terms of the first column represent the number of ICs that belonged to A but were classified as either B ( $1^{\text {st }}$ off-diagonal term) or C ( $2^{\text {nd }}$ off-diagonal term), each divided by the total number of ICs that belonged to A. Hence, the colour bar in this figure portrays the fraction of correctly (diagonal) or incorrectly (off-diagonal) classified ICs from the total number of ICs in one class (read column-wise). This decision was based on the maximum posterior probabilities across the three classes (from Figure 3). Therefore, a confusion map summarises the variations that the independent components' amplitude or phase 
undergo in one frequency band of interest during the three states (Task-ER, Task-Background and Rest).

Since Neuroscale and GMMs provide a generative model of the training data points onto a 2-D feature space (rather than a simple mapping), a new batch of recordings can be treated as a test dataset (Low and Tipping, 1996; Nabney, 2004); their features (obtained by following Stages (I) and (II)) can be projected onto the output of the training system (from Stage (III)) and the classification probabilities derived accordingly (Stage (IV)).

\subsection{Datasets for the Overall Results}

This multistage methodology was employed on the data of 20 out of the 23 participants in this study. The data from two participants were unusable due to poor quality EEG recordings (these datasets contained gross artifact laden channels, the poor quality of which persisted even after ICA artifact removal, hence the data was deemed unreliable), whilst one participant was unable to perform the tasks correctly (obtaining a $78.5 \%$ and $88 \%$ error rate during Go No-go and the OB Hard task respectively, and reported impatience and anxiety during the rest periods). Such performance would create outliers in the otherwise normal population and could potentially skew the final results; thus this participant's data was excluded from the analysis. For each of the three task environments, the remaining 20 participants were split into a training dataset (14 participants) and a test dataset (6 participants). The participants were divided in this way to ensure that the training set was comparatively larger that the test set, an approach suggested to both ensure model robustness and give good generalizability to new data (Hastie et al., 2009). For more information regarding this dataset refer to Appendix.

\section{Results}

\subsection{Performance Measures}

For the SART, the error rates were derived from the number of false alarms, i.e. button presses to Nogo responses; the number of misses to Go's was negligible. Across task blocks and participants the overall average RT and error rates were $(441 \pm 58) \mathrm{ms}$ and (mean: $25.4 \%$, range: [4.6 48.2]) \% respectively.

For the OB Task, the mean RT, percentage error rate: misses to targets, and percentage of false alarms: hits to standard stimuli (since none of the participants responded to distractors) were computed for the Easy and Hard task blocks consecutively. Since the data were not normally distributed a Wilcoxon signed-rank test was used for comparison between the Easy and Hard task conditions. Results show

that for the Easy task condition RTs were significantly shorter, and error rates and percentage of false alarms significantly smaller in comparison to the Hard task condition (Supplementary Table 1). 


\subsection{P300 Responses}

Preliminary analysis investigated the P300 responses during the three task environments for the 20 participants. TDSEP ICA successfully extracted components associated with the three types of P300 responses - these were ICs whose coherent average showed a clear peak around 300ms after stimulus onset, and whose topography was associated with that expected for P300 responses elicited by the task at hand, as shown in Figure 5(a). A robust P300 with a central distribution was elicited during the SART, as shown in (i); during the OB task, P300s with a frontal topography were elicited in response to distractor stimuli, as shown in (ii); target stimuli generated P300s with a parietal focus, as shown in (iii).

For the SART, the No-go responses exhibited significantly higher amplitudes (measured by considering the largest positive-going peak relative to the pre-stimulus baseline within a latency window of 300 to $600 \mathrm{~ms}$ ), and longer peak latencies (measured from the time of stimulus onset) on centro-parietal channels than the go responses, (e.g. Pz amplitude: $7.68 \pm 2.95$ vs $4.01 \pm 1.57 \mu \mathrm{V}$, $\mathrm{t}(1,19)=4.54, \quad p$-value=0.0014; latency: $450 \pm 43$ vs $361 \pm 11 \mathrm{~ms}, \mathrm{t}(1,19)=6.18, p$-value $<0.001)$. The grand coherent average responses across the 20 participants, and across both task blocks for channels $\mathrm{Fz}, \mathrm{Cz}$ and $\mathrm{Pz}$, are illustrated in Figure 5 (b).

For the OB Task, the group P300 responses elicited by the target, distractor and standard stimuli during the Easy and Hard task conditions for channels Pz and Fz are illustrated in Figure 5 (c). During the Easy task, the P300 elicited by the target, had a significantly higher amplitude in centro-parietal areas (Pz: $8.85 \pm 3.67$ vs $5.95 \pm 3.24 \mu \mathrm{V}, t(1,19)=5.31, p$-value $<0.001$ ) and significantly shorter latency (Pz: $481 \pm 49.78$ vs $517 \pm 57.55 \mathrm{~ms}, t(1,19)=-2.61, p$-value=0.0287) in comparison to the Hard task condition. Meanwhile, the more frontal P300 component elicited by the distractor stimuli, showed significantly higher amplitude during the Hard task (Fz: $5.10 \pm 2.40$ vs $2.79 \pm 1.79, t(1,19)=5.49, p$ value $<0.001$ ), whereas its latency varied very little across the two conditions ( $p$-value $>0.1)$. No significant difference was found for P300 amplitude or latency elicited by the frequently occurring standard stimuli in both task conditions.

\subsection{Multistage System Analysis Results: Training Data}

Figure 6 (a) shows an example of the classification results based on the ICs amplitude features when filtered in the slow wave band during the OB Hard task environment for the training databank. Note the clear distinction between the ER and Background ICs features and the overlap between Rest and Task classes. Figure 6 (b)-(d) then shows training data classification results based on delta, theta and alpha features respectively for selected task environments (similar patterns were obtained for all three task environments; for more results see Supplementary Figure S2). 
The sensitivity of classification for the training databank for the three task environments when using the characteristics of the amplitude or phase of the ICs in the four frequency bands is illustrated in Figure 7(a) and (b), first column. The slow waves experience the highest level of overlap (lowest sensitivity) between the rest and task classes, whilst achieving the highest separation for the Task-ER and Task-Background classes in comparison to the other oscillators. Moreover, for the slow wave band the sensitivity of classification for the Rest improves as the task difficulty increases from SART to OB Hard, (Slow wave amplitude features: SART - 31.1\%, OB Easy - 38.3\%, OB Hard - 43.6\%; Slow wave phase features: SART $-32.4 \%$, OB Easy $-42.1 \%$, OB Hard $-46.1 \%$ ).

Finally, in order to get an overall picture of how the four oscillators were changing together during rest and task, the AR models of the amplitude of the four $f$-bands were concatenated and used in the classification method. The results shown in Supplementary Figure S3 demonstrate a high sensitivity of classification for the three classes even for the easiest of the three task environments (SART).

\subsection{Multistage System Analysis Results: Test Data}

As previously explained, the test databank containing the ICs features of the remaining 6 participants was then mapped onto the 2-D feature space provided by the neuroscale output for the corresponding training databank (i.e. for a particular task environment and frequency band). The PDFs of the 2-D output mapping were then characterised by projection onto the three corresponding training GMMs. Test data results for the sensitivity of classification are summarised in Figure 7(a) and (b), second column.

\subsection{Slow Waves Power}

Classification results demonstrate that by using only the slow wave amplitude or phase features one can distinguish between three distinct types of brain activity. Hence, the slow waves experience considerable change with a switch from task to rest. To further characterise these changes, the slow wave power (the sum of the amplitude squared) was calculated for the Task ICs (ER and Background taken together) and the Rest ICs when filtered in the slow wave band, for all 20 participants and separately for each task environment. The average slow wave power during task was always lower in comparison to that during rest: $58.6 \%$ reduction ( $p$-value $<0.0001, \mathrm{z}=4.46$ ) exhibited during the SART, $46.2 \%$ reduction for OB Easy ( $p$-value $<0.0001, z=6.35$ ) and $35.9 \%$ reduction for the OB Hard task environment ( $p$-value $<0.0001, \mathrm{z}=5.92$ ). An unbalanced two-way ANOVA with paradigm (SART, OB Easy, OB Hard) as one factor and condition (Rest, Task) as another factor showed significant main effect of factors ( $p$-values<0.0001), as well as significant interaction between the two ( $p$ value $=0.0389$ ). Post-hoc (Bonferroni correction) comparison analysis revealed that the attenuation 
effect was higher for the SART in comparison with the OB Easy and OB Hard tasks, whilst the difference between OB Easy and OB Hard power did not reach statistical significance.

\subsection{Investigating the Relationship between Classification Results and Behavioural Data}

The sensitivity values of the classification results obtained from the training data, based on bandlimited IC features for different task environments, were correlated with behavioural data, namely reaction time and error rates. This was done in order to assess whether the difference between the three brain states could be linked with performance throughout the course of the task. Recall that a high sensitivity of classification indicates that the relative change in the amplitude or phase features between ICs related to task processing (ER), the Background ICs during task and those during Rest, is significant enough for them to be classified separately. Significant correlation results are shown in Figure 8. Note that the training dataset has been used for this purpose, since its classification results were more robust, and more data points were available for computing the correlation coefficients and $p$-values.

\section{Discussion}

In this work we present a new experimental framework and a multistage signal processing methodology for investigating the measurable effects of the intrinsic slow waves $(f \leq 0.5 \mathrm{~Hz})$ in brain activity during periods of quiet wakefulness, and whilst performing goal-directed tasks of various difficulty levels. This study explored the functional relationship between slow waves recorded at rest and those following the transition to task-specific attention and performance. In these experiments, the brain was conceptualised as a system comprising a number of oscillators of varying frequencies (Rothenberger, 2009), onto which a graded load was imposed to yield a proportional output response the P300. Here we set out to address the following questions: (i) What are the core differences between the slow waves recorded at rest and those undergoing cognitive load during task-specific attention? (ii) How are the slow waves affected by different levels of cognitive load and therefore requirements on task-related attention? Furthermore, we have also considered higher, more conventional frequency bands, namely delta, theta and alpha, for comparison with the slow waves.

\section{ERPs and performance measures}

The experimental protocols adopted by the current study successfully elicited typical P300 responses, the amplitude and latency of which were sensitive to manipulations of cognitive load. The robust Go No-go P300's shown in Figure 5(b) and the performance measures indicate a good level of attention throughout the course of this task for most participants. As expected, the No-go stimuli, with $12 \%$ probability of occurrence, evoked significantly larger P300 amplitudes in central and centro-parietal brain regions, a result that is in keeping with the literature (Katayama and Polich, 1996; Polich, 2007). 
During the oddball task, significantly longer reaction times, and significantly higher error and false alarm rates were observed during the Hard task condition (when the target was similar to the standard stimulus) in comparison to the Easy condition. This is because small standard-target perceptual differences demand more stimulus processing as previously shown in the literature (Comerchero and Polich, 1999; Hagen et al. 2006). Moreover, the OB P300 responses of Figure 5 (c) show a clear P300 subcomponent (termed as the P3b) elicited by the target at the parietal channel Pz, with a significantly higher amplitude and shorter latency for the Easy task condition. This supports earlier works which show that increased task difficulty recruits more sensory and working memory functions, yields longer stimulus evaluation time, and results in amplitude reduction and longer peak latency for the P3b - this subcomponent represents the executive control function, focused attention (Bledowski et al., 2004; Polich, 2007). Increased task difficulty also appears to engage frontal attentional mechanisms more strongly, producing a large frontal/central P300 component (also termed the P3a subcomponent of the P300) when a distracting stimulus interrupts attentional control (Comerchero and Polich, 1998; Hagen et al., 2006). This is shown in Figure 5 (c), where the frontal P3a component at channel $\mathrm{Fz}$ has significantly higher amplitude for the Hard task condition. The latter suggests that under higher cognitive load (increased task-related attention) the distractor captured attention more readily than in the Easy task, where less overall attention was required to differentiate the target from the standard stimulus, thus enhancing P3a responses. These results substantiate the findings by Comerchero and Polich (1999), and Hagen et al. (2006), and confirm the successful manipulation of graded task difficulty in the experimental protocol, as well as proper task execution throughout.

\section{Classification results}

Classification results from our multistage system showed consistent variations between the independent components during ER and Background task activity, and during Rest for all four oscillators (Figure 7). Particularly, using only the slow wave amplitude or phase features one can distinguish between the three distinct types of brain activity, and the sensitivity of classification is enhanced for higher task difficulty (from SART to Oddball Hard). Moreover, both the amplitude and phase of the ER and Background task ICs were very different in the slow wave band (Figure 6 (a)) this could be due to the different morphology of these two types of task ICs. Meanwhile, the substantial overlap between rest and task classes in the slow wave band, in comparison to that observed for higher frequencies such as theta and alpha, is consistent with the VLF theoretical models and fMRI BOLD imaging studies on the slow waves (Fox et al. 2006; Fransson, 2006; Fox and Raichle, 2007; Sonuga Barke and Castellanos, 2007; Deco, 2011), which suggest that despite experiencing changes during different brain states the slow waves persist across these states. The slow wave mechanism that is affecting the brain sources and/or that is being affected by the different loadings during task does not operate in a binary fashion, i.e. it is not naturally turned "on" and "off" following a rest-to-task transition. Rather the ongoing slow wave activity might be causing the resting 
brain to operate at the edge of bifurcation, awaiting the next cognitive act (Deco, 2011). Furthermore, the rest-to-task separation of the slow wave features, although low, might be reflecting the different VLFOs' relationships such as those exhibited by task positive and task negative networks (Fox et al., 2005).

Although, as expected, the test data sensitivity values were lower than those obtained for the training data (Figure 7), the two sets of results are comparable; for example, using the slow wave features, the ER and Background classes can be separated with the highest sensitivity in comparison to the other oscillators. Moreover, the test data also demonstrates a rise in the slow wave sensitivity (i.e. in the percentage of correct classification) for the Rest class as the task difficulty level is increased. This implies that the amplitude and phase in the slow wave band are undergoing enhanced changes between rest and task with increased cognitive load, which demonstrates their sensitivity to external stimulation albeit their persistent activity throughout.

Meanwhile, the distinctive separability between rest and task for the higher, more conventional frequency bands (shown in Figure 6(b)-(d) and Figure 7), particularly the alpha $f$-band, comes as no surprise and has been well acknowledged in the literature (Herrmann and Knight, 2001; Ben-Simon et al., 2008). The alpha oscillator is believed to act as a "stand-by" state or a "self-resonance" system that allows the brain to return more rapidly to goal-oriented cognitive functioning if and when required (Herrmann and Knight, 2001). When the primary visual cortex receives no or little input, it oscillates predominantly in the alpha range at a relatively high amplitude. In fact, as seen from these experiments, the most prominent activity in the EEG at rest is the ongoing alpha oscillatory component, which is thought to reflect cortical idling. This activity is desynchronised by the presence of stimulation and the total alpha power is decreased (Herrmann and Knight, 2001). Furthermore, stimulation resets the randomly distributed phase of the alpha waves, leading to increased phaselocked alpha activity. This explains the very high sensitivity of classification for the Rest class during all task environments, for both amplitude and phase ICs features in the alpha $f$-band.

\section{Slow wave power and the putative link to attention}

The slow waves in the resting brain exhibit attenuation in their power during task execution as predicted by the DMI hypothesis. Consistent with early fMRI evidence, the DMI hypothesis also predicts greater rest-task attenuation with increasing task difficulty or cognitive load. For example, using a visual detection task, Singh and Fawcett (2008) demonstrated a linear increase in the deactivation of DMN regions with increasing task difficulty. McKiernan et al., (2006) also found that corresponding increases in task-related deactivation of DMN regions and task difficulty were associated with reductions in a measure of mind-wandering. In the current study, the SART was found to elicit greater task-related attenuation of VLFOs when compared with either the Easy or Hard 
OB task conditions, which were not found to differ significantly in terms of the degree to which VLF power was attenuated during the transition to task-related attention. This finding could indicate that the SART might have been more taxing for the brain than initially intended (as a simple, repetitive task). These two tasks may also recruit different executive functions to different degrees (e.g. attention during the oddball task but inhibition during SART), which makes their comparison harder. Moreover, a higher task-induced deactivation (demonstrated as a local decrease in blood flow during an active task relative to a "resting" baseline) observed with increased task difficulty (McKiernan et al., 2006; Singh and Fawcett 2008), might not necessarily implicate a greater reduction in VLF EEG power, since BOLD and EEG signals are not one-to-one representations of neural activity. Also, it has recently been shown that some types of slow EEG activity correlate positively with increasing task load (Michels et al., 2010). Future research should replicate these findings with a greater range of attention-demanding tasks and task conditions.

Furthermore, the level of cognitive attention (assessed by means of performance measures, namely reaction time and error rates) exhibited significant correlations with the changes that the slow waves underwent between rest and task (assessed through the sensitivity of classification based on the slow wave features) showing a significant link between these slow electrical oscillators and cognitive attention (Figure 8). In the slow wave band, significant correlations were found for the Oddball task and not for the SART. This correlation tentatively suggests that the better the participants' performance (i.e. the lower the reaction time and error rates) the greater was the difference in the slow wave band between, for instance, the Task-ER and the other (Task-Background and Rest) brain activity, hence the higher the sensitivity of classification. We remark that although the Oddball Hard task slow waves amplitude features provide good classification performance, the negative-correlation with behavioral data did not reach statistical significance, despite showing the same trend (e.g. RT vs Correct ER: $R=-0.43, p$-value=0.09; Error Rate vs Correct ER: $R=-0.46, p$-value=0.1). It is important to note here that the main aim of this study has been to investigate the slow waves, and despite the long recordings that have been used to obtain the classification results (which makes them robust), the number of participants (and hence the number of available performance data points) is small to perform reliable statistics on. Moreover, the work has been performed on a normal, adult population. This implies that the interpretation of these correlation results needs to be made with caution, and the whole procedure merits further investigation using a case-control design in a population with higher levels of behavioral variability such as ADHD. Such data would provide useful information about the relationship between brain electrical oscillations and behaviour, a link that has been explored in the works by Fox et al. (2007), Monto et al., (2008) and Helps et al. (2008), and is fundamental for advances in the assessment of child psychiatric disorders (Rothenberger, 2009). 


\section{Slow waves origin and function}

In past decades, other studies have looked at DC-EEG activity from the perspective of slow and sustained negative DC potential shifts. These slow shifts have been recorded over the scalp during a variety of cognitive (Lang et al., 1992) and motor (Lang et al., 1988) tasks. For example, they have been shown to contain task specific patterns, their scalp distributions reflect differences in cortical areas activated during tasks, and they are sensitive to task characteristics such as stimulus modality (e.g. visual/auditory, Lang et al., 1992), and type of movement (e.g. sequential/simultaneous finger movements, Lang et al., 1988). In the latter study, the amplitude of the slow Bereitschaftspotential on central midline regions preceding the voluntary initiation of a motor task has been shown to increase with task difficulty. Thus, these large slow potential shifts (which do not necessarily show regular periodicity) may share some characteristics of the VLFOs described in this work (as suggested also in the review by Vanhatalo et al., 2005). Whilst it is thought that these scalp-recorded DC potential shifts reflect depolarization of layers I and II of atypical dendrites of vertically oriented pyramidal cells (Lang et al., 1992), the origin of the VLFOs remain as yet unclear. Some literature proposes that VLFOs may arise from periodic increasing and decreasing of the neurons' firing (say every 10 seconds) in the midbrain reticular formation (Balduzzi et al., 2008). Another possibility is that neurons slowly modulate their level of activity because of intrinsic variations in ionic pumps, neurotransmitter transporters and glial cells (Balduzzi et al., 2008; He et al., 2008). Meanwhile, these oscillations may be essential for the development and organization of neuronal systems (Fox et al., 2006); alternatively they could be emerging from the network(s) itself rather than from the individual neurons (He et al., 2008). Furthermore, VLFOs may represent dynamic modulations in the internal brain representation, providing a basis onto which perception and behaviour occur, and hence determining our response to the outside world. Since this intrinsic brain activity is observed in both goal-oriented tasks and rest, it may represent continuous processes which are vital for maintaining a coherent neuronal representation of the 'self' (Fransson, 2006). Our work, in keeping with the evidence in the literature (Laufs et al., 2003; Leistner et al. 2007; Monto et al., 2008) promotes the view that the slow waves, spontaneous or evoked, are real neuronal responses.

\section{The multistage system}

Lastly, although most of the stages in our multistage system are based on standard signal processing methods which have been around for years, at first glance the whole procedure may seem overly laborious and complicated. Hence, it is important to outline the main reasons why we opted for this particular experimental set-up and data analysis methods, and the potential benefits of such an approach. First, VLF brain activity is notoriously difficult to study; one must ensure that the observations made arise from neuronal activity and are not due to other physiological (respiratory, cardiac and vasomotion) processes (Vanhatalo et al., 2003; Voipio et al., 2003, Auer, 2008), and/or arise from inherent properties in the data (such as the $1 / f$ spectral trend of the EEG; Demanuele et al., 
2007), or from the data analysis procedure itself. Hence both the analysis and interpretation of slow signals related to cognitive tasks needs to be done with caution. One way to ensure this is to adopt an experimental set-up that enables us to compare the VLF EEG under different levels of stimulation, whilst keeping everything else constant (same participants and same analysis methods). Other paradigms such as working memory tasks which manipulate memory load (Pyka et al., 2009), and simple choice reaction time attention tasks (Helps et al., 2009:2010) could also be implemented and the oscillations contrasted with the same methodology. It would be interesting to include experimental tasks with more frequent sampling (i.e. short inter-stimulus intervals) to facilitate a near continuous performance measure of attention which might be examined concurrently with EEG recordings. Further, the two experimental paradigms employed in the current study, the SART and OB task, allowed participants to make errors of omission and commission, and included multiple stimulus types with differing performance requirements. To examine the association between these VLF brain oscillations and performance measures of attention (such as RT variability) more precisely, future research should also include tasks which do not involve multiple stimuli and do not allow participants to omit or make incorrect responses.

Second, a straightforward approach for investigating this VLF activity is to specifically analyse the raw data in the frequency range below $0.5 \mathrm{~Hz}$ by standard Fourier-domain based methods (Bruns, 2004). However, this approach proves to be very long and cumbersome to apply on multi-dimensional systems because the dense (high number of electrodes) and noisy nature of the recordings makes the interpretation of the results highly subjective. Denoising and demixing by proper filtering methods and/or by BSS techniques is definitely helpful. Moreover, BSS yields independent components which represent estimates of the underlying sources, hence enabling us to work on a sphere of influence between the scalp recordings and the true signal generators. The extracted components must then be subjectively identified as being artifactual, linked to the task at hand, or as mainly contributing to the background neuronal activity - hierarchical clustering based on their coherent average is one way to objectively achieve this grouping.

Third, the classification stages in our method, based on amplitude and phase features that describe the ICs, provide an objective method to deal with the comparative nature of the experimental set-up. The resulting confusion maps directly quantify the changes in one frequency band between two (or more) task conditions (e.g. ER, background and rest), hence enabling direct comparisons across paradigms and across frequency bands. In the case of the slow waves, this helps to put the results in perspective of the more common higher-frequency findings in the EEG literature, hence aiding their interpretation. It is worth noting here that direct comparison of the slow waves with higher frequency bands is not very straightforward; for example, the $1 / f$ intrinsic nature of the EEG power spectrum implies that the slow wave band will always contain higher power in comparison to other frequency bands (see 
Demanuele et al. 2007 for a method that tackles this). Furthermore, since evidence in the literature suggests that this spontaneous VLF activity might act as a 'baseline' that underlies brain activity elicited by the task (Fox and Raichle, 2007; Balduzzi et al., 2008; Monto et al., 2008; Demanuele et al., 2010), it may not be revealed as 'oscillations' in the classical sense of having peaks in the power spectra of the EEG channels per se, or as separate independent components following the demixing procedure. Using more sophisticated methods, such as the one presented here, could help unravel what might otherwise be obscured in the data, and could potentially lead to more insightful findings. Finally, the classification performance in different frequency bands could be used to compare bandlimited brain activity to performance measures (as attempted here), and/or to more subjective measures such as participants' reports on their level of attention or mood. This could be of considerable interest in studies involving clinical and control groups.

\section{Conclusion}

The current set of results and related methodology represent a novel approach to the analysis of VLFOs and provide new and important insight into the association between slow wave brain activity and conventional EEG frequency bands, demonstrating very clearly that VLF EEG oscillations are not physiological noise or a passive electrical phenomenon but real responses in the functioning brain. In keeping with recent models of VLF resting state brain activity, we show that VLFOs are attenuated but not extinguished following a transition from rest to task, and that they are modulated by variations in cognitive load (Sonuga-Barke \& Castellanos, 2007). Future work should attempt to clarify where in the brain these changes in VLF EEG are most prominent (e.g. Broyd et al., 2011). Furthermore, this novel experimental framework and signal processing methodology should also be explored with respect to altered functioning of brain oscillators in psychiatric and neurobehavioural disorders such as schizophrenia and ADHD (Broyd et al., 2009).

\section{Supplementary Material}

Supplementary material related to this article can be found online at: specify link. 


\section{References}

Auer AP. Spontaneous low-frequency blood oxygenation level-dependent fluctuations and functional connectivity analysis of the 'resting' brain. Magn Reson Imaging 2008;26:1055-1064.

Balduzzi D, Riedner BA, Tononi G. A BOLD window into brain waves. PNAS 2008;105:1564115642.

Başar E. Principles and approaches, brain function and oscillations: I. Brain oscillations. Heidelberg: Springer, 1998.

Başar E. Brain function and oscillations: II. Integrative brain function, neurophysiology and cognitive processes. Heidelberg: Springer, 1998.

Bear M, Connors B, Paradiso M. Neuroscience: Exploring the brain, $2^{\text {nd }}$ ed. USA: Lippincott Williams and Wilkins, 2001.

Ben-Simon E, Podlipsky I, Arieli A, Zhdanov A, Hendler T. Never resting brain: simultaneous representation of two alpha related processes in humans. PLoS ONE 2008;3:e3984.

Bishop CM. Neural networks for pattern recognition. New York: Oxford University Press Incorporation, 1995.

Biswal BB, Yetkin FZ, Haughton VM, Hyde JS. Functional connectivity in the motor cortex of resting human brain using echo-planar MRI. Magnet Reson Med 1995;34:537-541.

Bledowski C, Prvulovic D, Hoechstetter K, Scherg M, Wibral M, Goebel R, et al.. Localizing P300 generators in visual target and distractor processing: a combined event-related potential and functional magnetic resonance imaging study. J Neurosci 2004;24:9353-9360.

Broyd SJ, Demanuele C, Debener S, Helps S K, James CJ, Sonuga-Barke EJS. Default-mode brain dysfunction in mental disorders: A systematic review. Neurosci Biobehav Rev 2009;33:279-296.

Broyd SJ, Helps SK, Sonuga-Barke EJS. Attention-induced deactivations in very low frequency EEG oscillations: differential localisation according to ADHD symptom status. PLoS ONE 2011;6:e17325.

Bruns, A. Fourier-, Hilbert- and wavelet-based signal analysis: are they really different approaches? J Neurosci Methods 2004;137:321-332.

Buzsáki G, Draguhn A. Neuronal oscillations in cortical networks. Science 2004;304:1926-1929.

Comerchero MD, Polich J. P3a, perceptual distinctiveness and stimulus modality. Cognitive Brain Res 1998;7:41-48.

Comerchero MD, Polich J. P3a and P3b from typical auditory and visual stimuli. Clin Neurophysiol 1999;110:24-30.

Datta A, Cusack R, Hawkins K, Heutink J, Rorden C, Robertson IH, et al.. The P300 as a marker of waning attention and error propensity. Comput Intell Neurosci 2007;93968.

Deco G, Jirsa VK, McIntosh AR. Emerging concepts for the dynamical organization of resting-state activity in the brain. Nat Rev Neurosci 2011;12:43-56.

Demanuele C, James CJ, Sonuga-Barke EJS. Distinguishing low frequency oscillations within the $1 / f$ spectral behaviour of electromagnetic brain signals. Behav Brain Funct 2007;3.

Demanuele C, Capilla A, Pérez Hernández E, Sonuga-Barke EJS, James CJ. Trial-to-trial variability in evoked neural responses exhibit a very low frequency temporal signature; A Magnetoencephalography study. J Psychophysiol 2010;24:7-24. 
Demanuele C, James CJ, Sonuga-Barke EJS. Investigating the functional role of slow waves in brain signal recordings during rest and task conditions. Proc $5^{\text {th }}$ IEEE EMBS PGBIOMED, Oxford, UK, 2009;53-54.

Estrada E, Nazeran H, Behbehani K, Burk J, Lucas E. Itakura distance: a useful similarity measure between EEG and EOG signals in computer-aided classification of sleep stages. Proc $27^{\text {th }}$ IEEE EMBS, Shanghai, China, 2005;1189-1192.

Falkenstein M, Hoormann J, Hohnsbein J. ERP components in Go/Nogo tasks and their relation to inhibition. Acta Psychol 1999;101:267-291.

Fox MD, Snyder AZ, Vincent JL, Corbetta M, Van Essen DC, Raichle ME. The human brain is intrinsically organized into dynamic, anticorrelated functional networks. PNAS 2005;102:9673-9678.

Fox MD, Snyder AZ, Zacks JM, Raichle ME. Coherent spontaneous activity accounts for trial-to-trial variability in human evoked brain responses. Nat Neurosci 2006;9:23-25.

Fox MD, Raichle, ME. Spontaneous fluctuations in brain activity observed with functional magnetic resonance imaging. Nat Rev Neurosci 2007;8:700-711.

Fox MD, Snyder AZ, Vincent J, Raichle ME. Intrinsic fluctuations within cortical systems account for intertrial variability in human behavior. Neuron 2007;56:171-184.

Fransson P. Spontaneous low-frequency BOLD signal fluctuations: an fMRI investigation of the resting-state default mode of brain function hypothesis. Hum Brain Mapp 2005;26:15-29.

Fransson P. How default is the default mode of brain function? Further evidence from intrinsic BOLD signal fluctuations. Neuropsychologia 2006;44:2836-2845.

Hagen GF, Gatherwright JR, Lopez BA, Polich J. P3a from visual stimuli: task difficulty effects. Int J Psychophysiol 2006;59:8-14.

Hastie T, Tibshirani R, Friedman J. The elements of statistical learning: Prediction, inference and data mining, $2^{\text {nd }}$ ed. USA: Springer, 2009.

Hayes MH. Statistical digital signal processing and modeling. USA: Wiley, 1996.

He BJ, Snyder AZ, Zempel JM, Smyth MD, Raichle ME. Electrophysiological correlates of the brain's intrinsic large-scale functional architecture. PNAS 2008;105:16039-16044.

Helps S, James C, Debener S, Karl A, Sonuga-Barke EJS. Very low frequency EEG oscillations and the resting brain in young adults: a preliminary study of localization, stability and association with symptoms of inattention. J Neural Transm 2008;115:279-285.

Helps SK, Broyd SJ, James CJ, Karl A, Sonuga-Barke EJS. The attenuation of very low frequency brain oscillations in transitions from a rest state to active attention. J Psychophysiol 2009;23:191-198.

Helps SK, Broyd SJ, James CJ, Karl A, Chen W, Sonuga-Barke EJS. Altered spontaneous low frequency brain activity in Attention Deficit/Hyperactivity Disorder. Brain Res 2010;1322:134-143.

Herrmann CS, Knight RT. Mechanisms of human attention: event-related potentials and oscillations. Neurosci Biobehav Rev 2001;25:465-476.

Hyvärinen A. Fast and robust fixed-point algorithms for independent component analysis. IEEE Trans Neural Netw 1999;10:626-634.

Itakura F. Minimum prediction residual principle applied to speech recognition. IEEE Trans Acoust Speech Signal Process 1975;23:67-72. 
James CJ, Hesse CW. Independent component analysis for biomedical signals. Physiol Meas 2005;26:15-39.

Karakaş S, Erzengin OU, Başar E. The genesis of human event-related responses explained through the theory of oscillatory neural assemblies. Neurosci Lett 2000;285:45-48.

Katayama J, Polich J. P300, probability, and the three-tone paradigm. Electroencephalogr Clin Neurophysiol 1996;100:555-562.

Kong X, Goel V, Thakor N. Quantification of injury-related EEG signal changes using Itakura distance measure. Proc IEEE ICASSP, Detroit, MI, USA, 1995;2947-2950.

Lang W, Lang M, Uhl F, Koska C, Kornhuber A, Deecke L. Negative cortical DC shifts preceding and accompanying simultaneous and sequential finger movements. Exp Brain Res 1988;71:579-587.

Lang W, Starr A, Lang V, Lindinger G, Deecke L. Cortical DC potential shifts accompanying auditory and visual short-term memory. Electroencephalogr Clin Neurophysiol 1992;82:285-295.

Laufs H, Krakow K, Sterzer P, Eger E, Beyerle A, Salek-Haddadi A, et al. Electroencephalographic signatures of attentional and cognitive default modes in spontaneous brain activity at rest. PNAS 2003;100:11053-11058.

Leistner S, Sander T, Burghoff M, Curio G, Trahms L, Mackert B-M. Combined MEG and EEG methodology for non-invasive recording of infraslow activity in the human cortex. Clin Neurophysiol 2007;118:2774-2780.

Le Van Quyen M, Foucher J, Lachaux J-P, Rodriguez EO, Lutz A, Martinerie J, et al.. Comparison of Hilbert transform and wavelets methods for analysis of neural synchrony. J Neurosci Methods 2001;111:83-98.

Linn S. A new conceptual approach to teaching the interpretation of clinical tests. J Stat Educ 2004;12:1-9.

Lowe D. Novel "topographic" nonlinear feature extraction using radial basis functions for concentration coding in the 'artificial nose'. Proc $3^{\text {rd }}$ IEEE ICANN, London, UK, 1993;95-99.

Lowe D, Tipping ME. Feed-forward neural networks and topographic mappings for exploratory data analysis. Neural Comput Appl 1996;4:83-95.

Lowe D, Tipping ME. NeuroScale: Novel topographic feature extraction using RBF networks. In Mozer M, Jordan M, and Petsche T, editors. Advances in neural information processing systems vol. 9. Cambridge: MIT Press, 1997:543:549.

McKiernan KA, D'Angelo BR, Kaufman JN, Binder JR. Interrupting the "stream of consciousness": an fMRI investigation. Neuroimage 2006;29:1185-1191.

Michels L, Bucher K, Lüchinger R, Klaver P, Martin E, Jeanmonod D, et al. Simultaneous EEG-fMRI during a working memory task: Modulations in low and high frequency bands. PLOS One 2010;5:e10298.

Monto S, Palva S, Voipio J, Palva JM. Very slow EEG fluctuations predict the dynamics of stimulus detection and oscillation amplitudes in humans. J Neurosci 2008;28:8268-8272.

Nabney IT. NETLAB algorithms for pattern recognition. London: Springer, 2004.

Penttonen M, Buzsáki G. Natural logarithmic relationship between brain oscillators. Thalamus Relat Syst 2003;2:145-152. 
Polich J. Updating P300: An integrative theory of P3a and P3b. Clin Neurophysiol 2007;118:21282148.

Pyka M, Beckmann CF, Schöning S, Hauke S, Heider D, Kugel H, et al. Impact of working memory load on fMRI resting state pattern in subsequent resting phases. PLoS ONE 2009;4:e7198.

Rothenberger A. Brain oscillations forever - neurophysiology in future research of child psychiatric problems. J Child Psychol Psychiatry 2009;50:79-86.

Shibasaki H, Barrett G, Halliday E, Halliday AM. Components of the movement-related cortical potential and their scalp topography. Electroencephalogr Clin Neurophysiol 1980;49:213-226.

Singh KD, Fawcett IP. Transient and linearly graded deactivation of the human default-mode network by a visual detection task. Neuroimage 2008;41:100-112.

Smallwood J, Beach E, Schooler JW, Handy TC. Going AWOL in the brain: Mind wandering reduces cortical analysis of external events. J Cogn Neurosci 2007;20:458-469.

Sonuga-Barke EJS, Castellanos FX. Spontaneous attentional fluctuations in impaired states and pathological conditions: a neurobiological hypothesis. Neurosci Biobehav Rev 2007;31:977-986.

Steriade M, Nunez A, Amzica F. A novel slow ( $<1 \mathrm{~Hz}$ ) oscillation of neocortical neurons in vivo: depolarizing and hyperpolarizing components. Neuroscience 1993;13:3252-3265.

Varela F, Lachaux J-P, Rodriguez E, Martinerie J. The brainweb: Phase synchronisation and largescale integration. Nat Rev Neurosci 2001;2:228-239.

Vanhatalo S, Voipio J, Dewaraja A, Holmes MD, Miller JW. Topography and elimination of slow EEG responses related to tongue movements. Neuroimage 2003;20:1419-1423.

Vanhatalo S, Palva JM, Holmes MD, Miller JW, Voipio J, Kaila K. Infraslow oscillations modulate excitability and interictal epileptic activity in the human cortex during sleep. PNAS 2004;101:50535057.

Vanhatalo S, Voipio J, Kaila K. Full-band EEG (FbEEG): An emerging standard in electroencephalography. Clin Neurophysiol 2005;116:1-8.

Voipio J, Tallgren P, Heinonen E, Vanhatalo S, Kaila K. Ultraslow DC shifts in the human EEG: evidence for a non-neuronal generator. J Neurophysiol 2003;89:2208-14.

Ziehe A, Müller KR. TDSEP - an efficient algorithm for blind separation using time structure. Proc $8^{\text {th }}$ IEEE ICANN, Skövde, Sweden, 1998;675-680. 


\section{Legends}

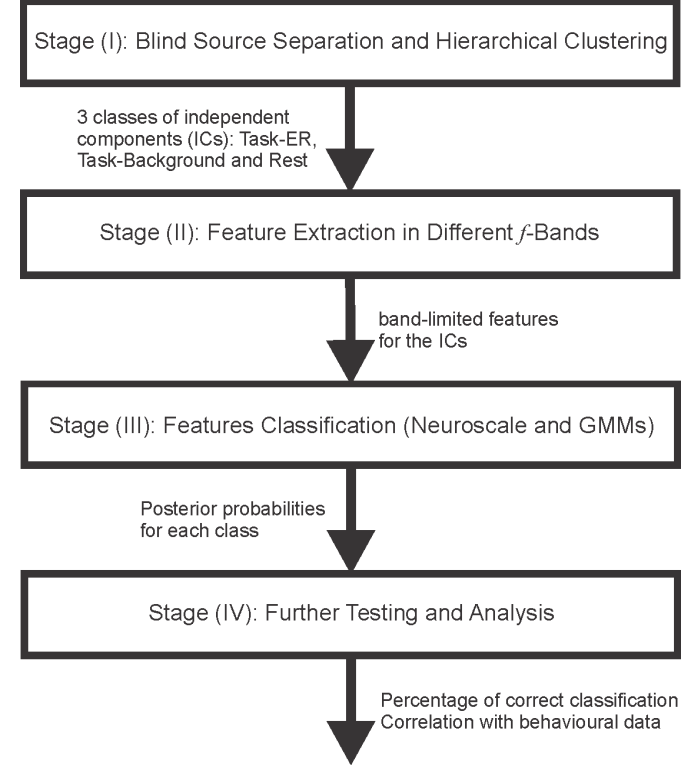

Fig. 1 - The multistage signal processing methodology developed for assessing variations in bandlimited oscillators in the EEG at varying levels of cognitive load, as well as their behavioural implications. 
(a) (i)

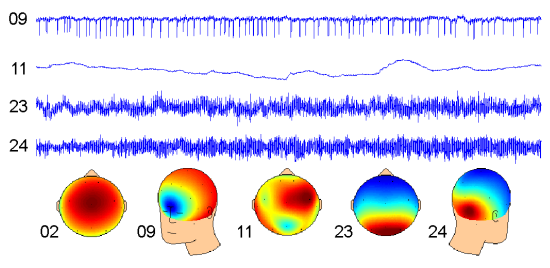

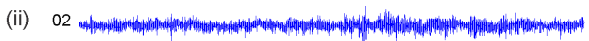

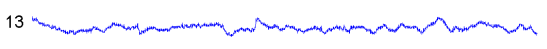

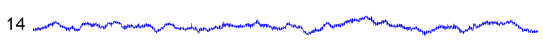

20 istity

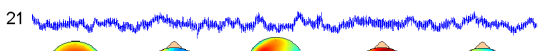

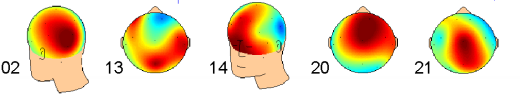

(c)

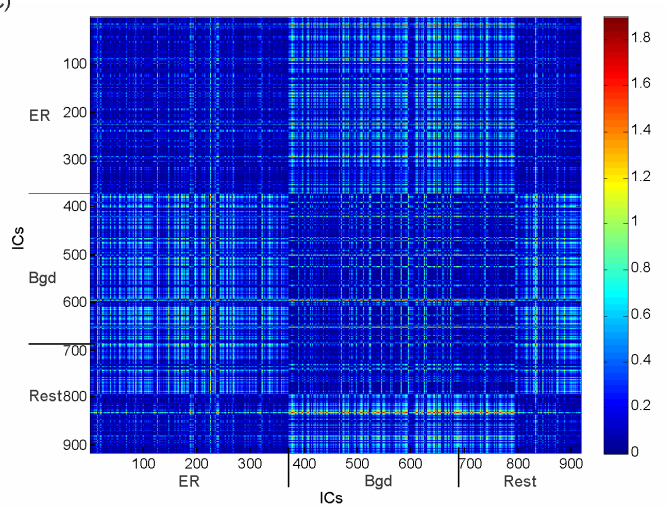

(e)

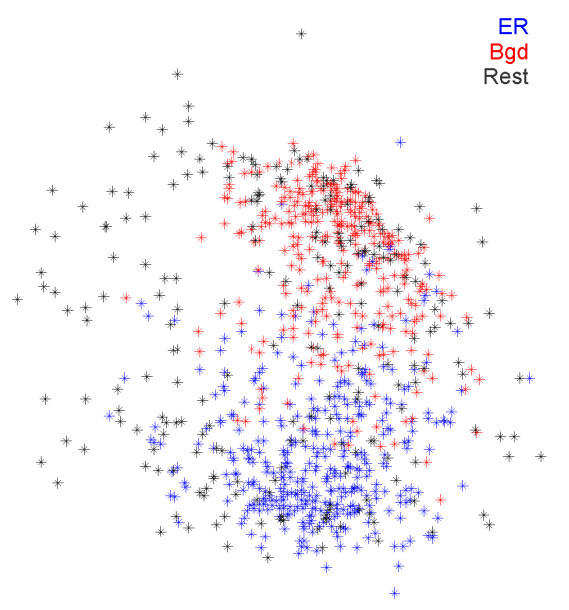

(b)

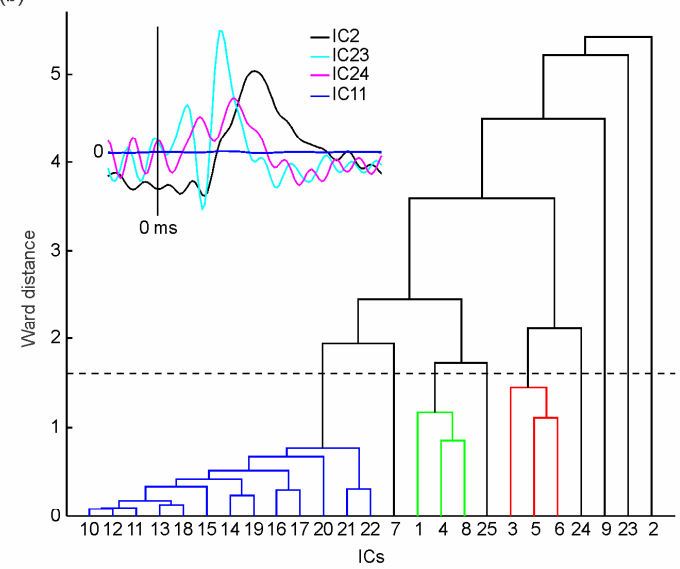

(d)

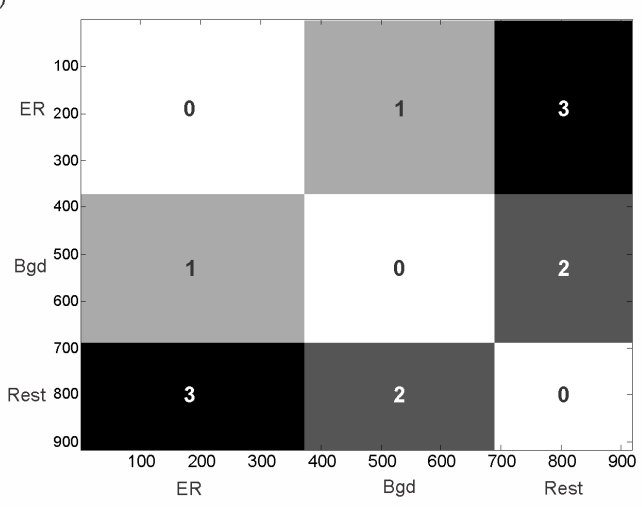

(f)

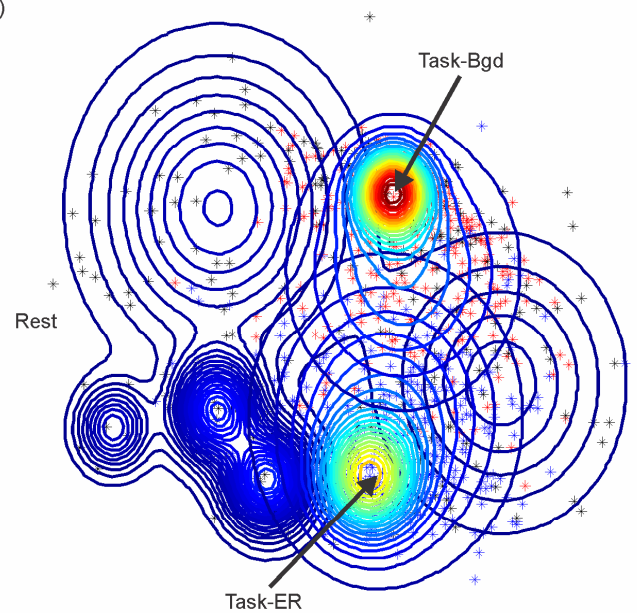

Fig. 2 - Analysis stages. (a) TDSEP-ICA decomposition (results of 1 participant shown as an example): Five of the 25 ICs and their spatial distribution extracted by TDSEP-ICA during (i) task and (ii) rest. (b) Hierarchical Clustering: dendrogram built on the coherent averages of the task ICs (selected traces shown): blue cluster corresponds to the background ICs, the red and green clusters and the individual branches correspond to the large ER-ICs, with the exception of IC9 (eye blinks), (Demanuele et al., 2009). (c) Scaled image of the ID between the AR models of the ICs slow wave 
instantaneous amplitude for the Oddball Hard task environment. Note that points 1:371 represent the ER class, 372:687 the Background class and 688:917 the Rest class. (d) Scaled image of the distance matrix $s$ (i.e. the distance metric defined for each pair of points in the whole dataset) used in supervised neuroscale as a means of introducing subjective information in the training process. (e) The 2-D output from supervised neuroscale $(\alpha=0.5$, number of basis functions centres $=80$ ) based on the ID matrix (c) and s-matrix (d). Blue represents ER ICs features, red the Background features and black the Rest features; the axes scales are arbitrary. (f) The three GMMs derived for the 2-D neuroscale output. Note the distinct ER (modelling the blue points in (e)) and Background (modelling the red points) GMMs; the Rest GMM (modelling the black points) overlaps with both, but still occupies a partially-distinct space.
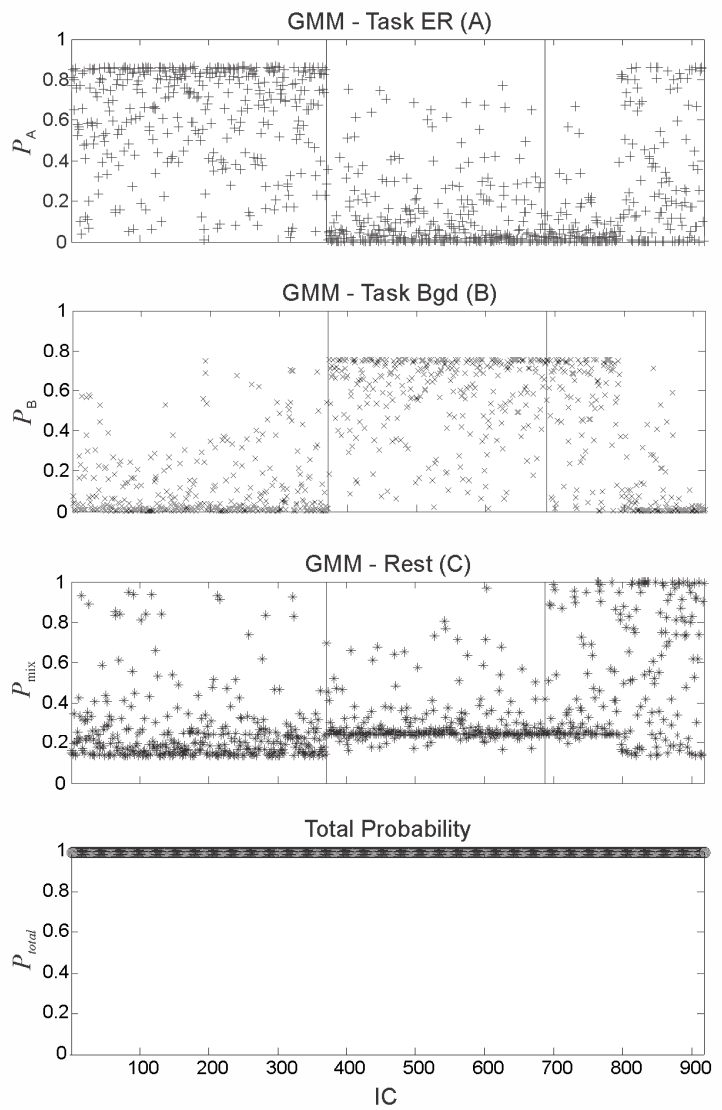

Fig. 3 - Normalised posterior probabilities for the 3 GMMs such that the probability for each data point adds up to one across the 3 classes. 


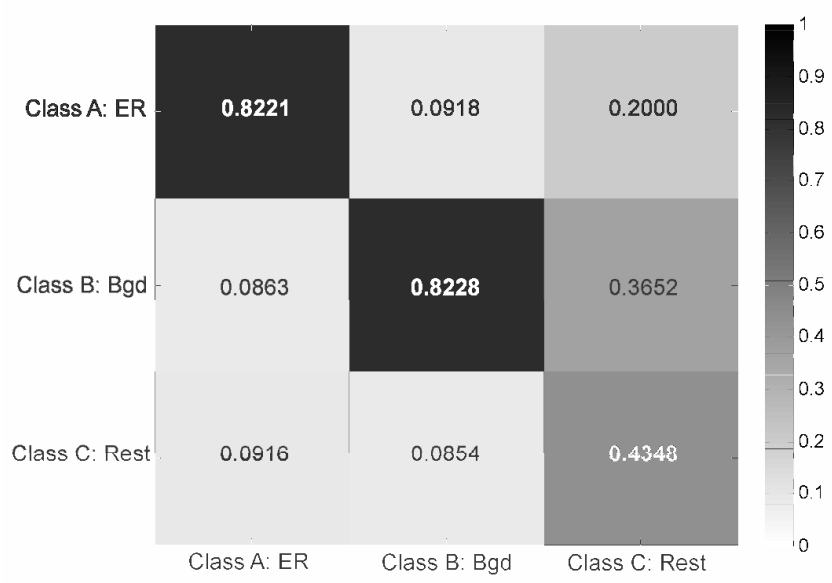

Fig. 4 - Confusion map for the classification based on slow wave amplitude ICs features. Diagonal values show the sensitivity of classification whereas the off-diagonal terms (column-wise) show the fraction of misclassified ICs for each class. 
(a) Examples of ICs associated with P300 responses for

(i) Go No-go
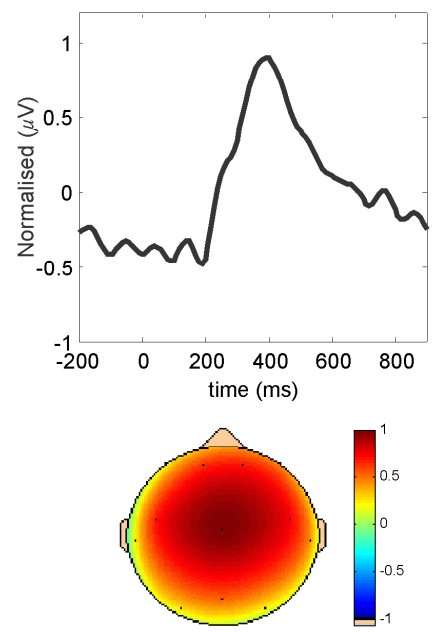

(b) Go No-go P300s

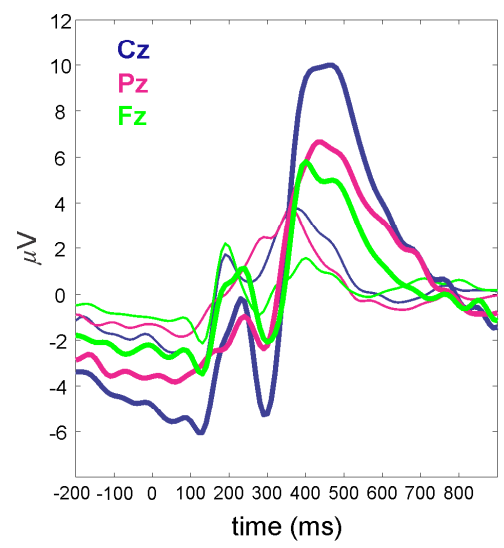

(ii) Oddball distractor
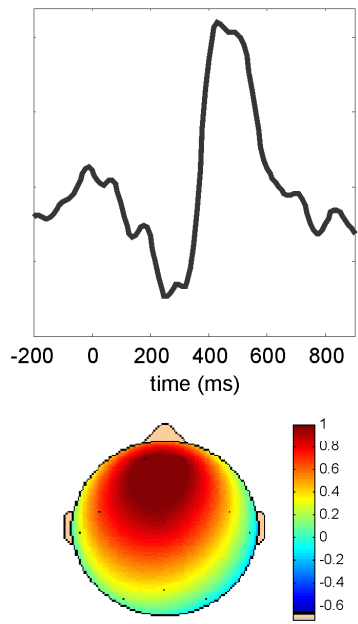

(iii) Oddball target
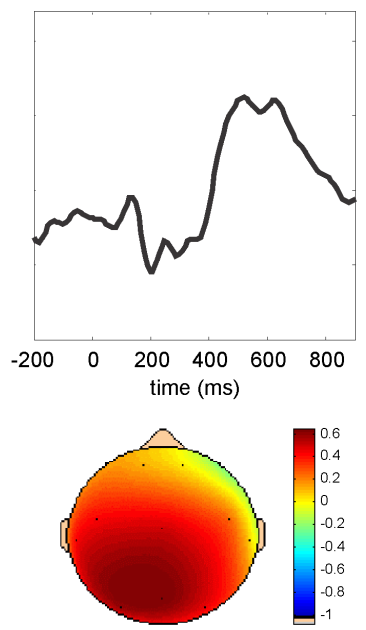

(c) Oddball Task P300s for Easy and Hard conditions

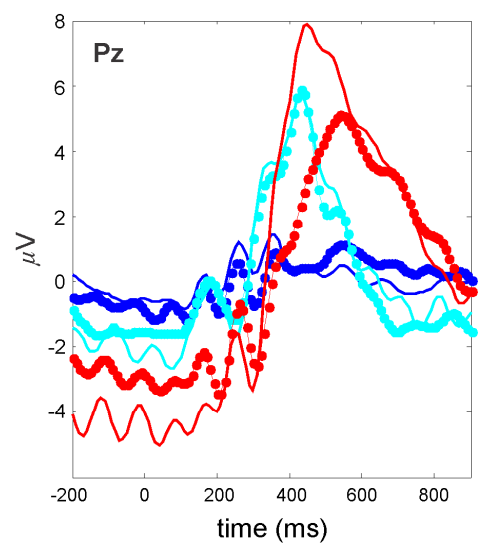

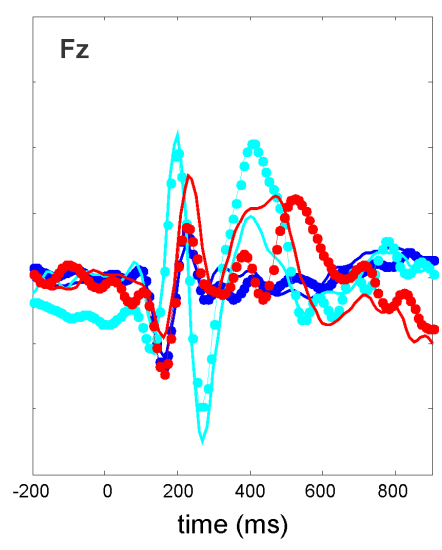

Fig. 5 - Event related potentials. (a) Three types of P300 responses (normalised amplitudes) extracted by TDSEP-ICA during the two tasks: (i) P300 with a central topography elicited during SART; (ii) P300 response with a frontal focus elicited by distractor stimuli; (iii) and P300 for target stimuli with a parietal focus, for the Oddball task. (b) Grand coherent average across the 20 participants and across the two task blocks, showing robust P300 responses during SART. (c) P300 responses during Oddball Easy and Hard task conditions for one parietal (strong target P300 response) and one frontal (strong distractor P300 response) channel. Standard stimuli P300s are shown in blue, distractors in cyan, targets in red; the solid and dotted curves correspond to the OB easy and hard tasks respectively. 
(a) Slow waves

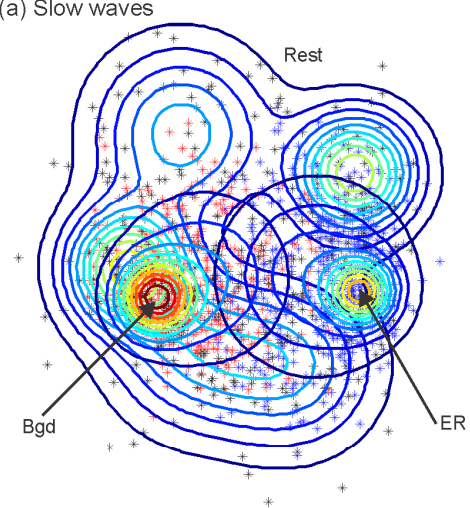

(b) Delta

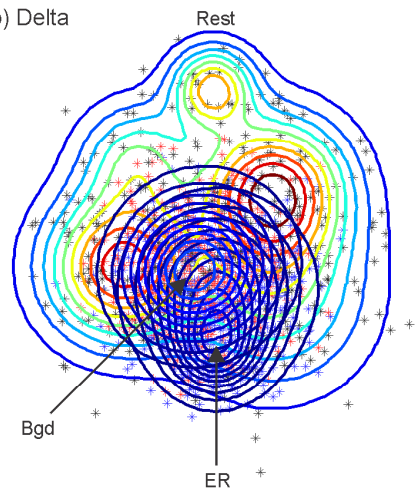

(c) Theta

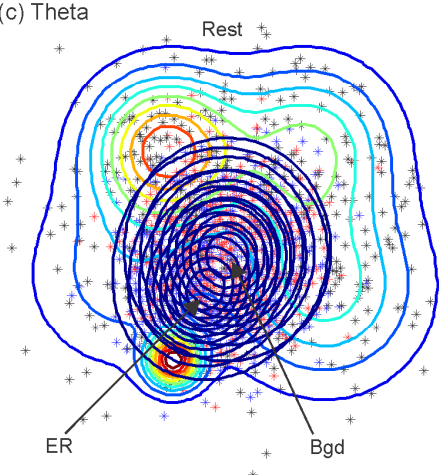

(d) Alpha

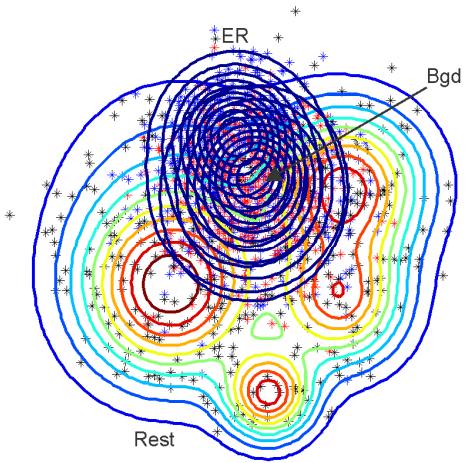

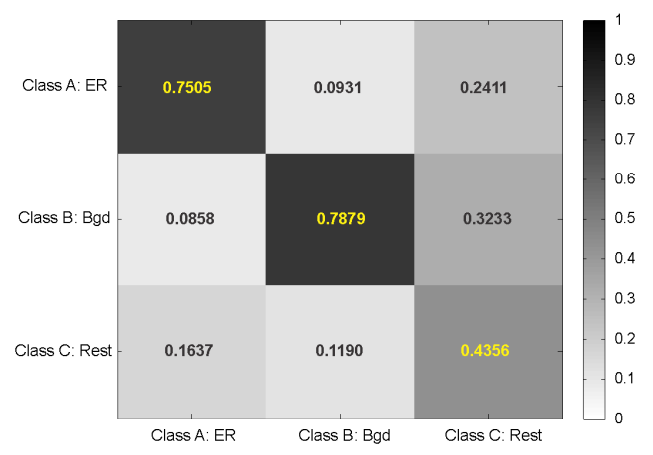
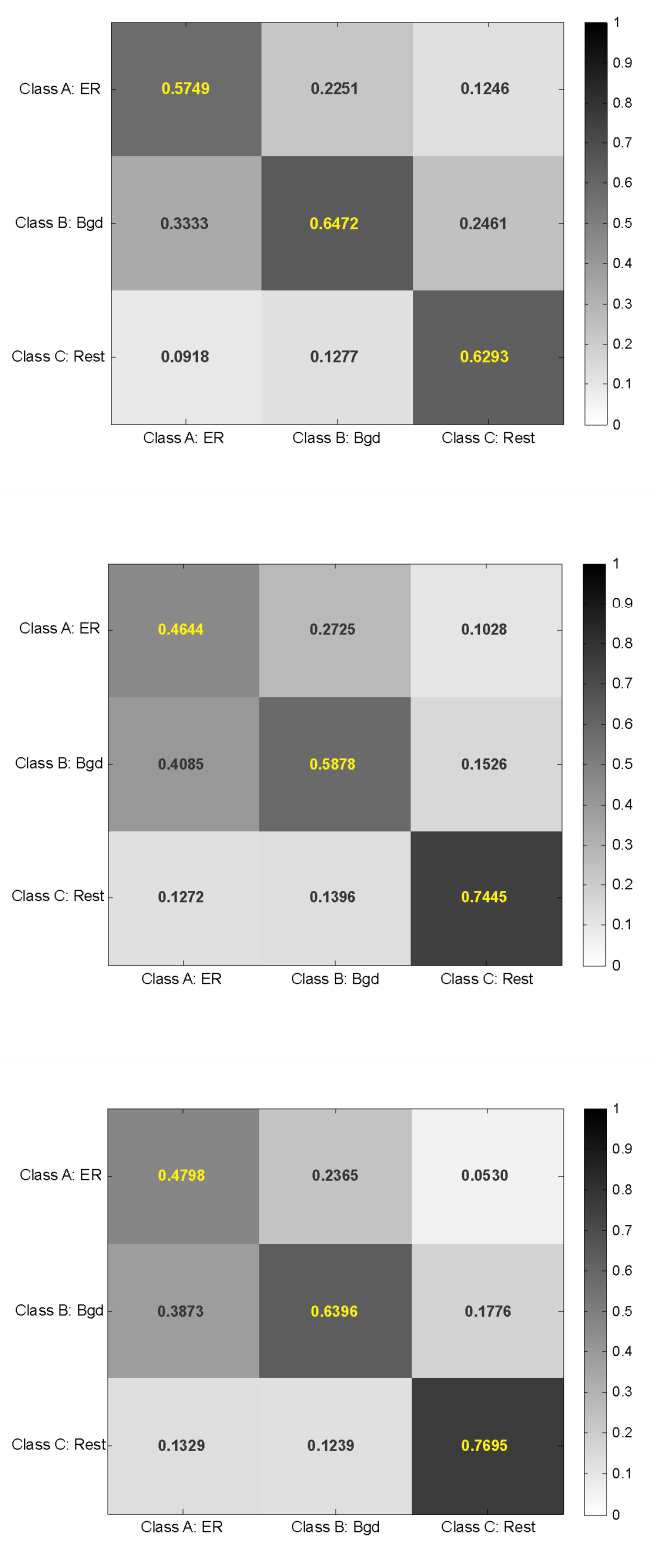

Fig. 6 - Training data classification results based on (a) Slow Wave Amplitude features and (b) Delta fBand Amplitude features of the ICs for the Oddball Hard task environment; (c) Theta f-Band Phase 
features and (d) Alpha f-Band Amplitude features of the ICs for the Oddball Easy task environment. $1^{\text {st }}$ column: GMM contour plots for the Neuroscale output. $2^{\text {nd }}$ column: Confusion map showing sensitivity of classification on the diagonal (derived from the GMMs posterior probabilities for the three classes as explained in text).

(a) Classification based on Amplitude features of the band-limited ICs
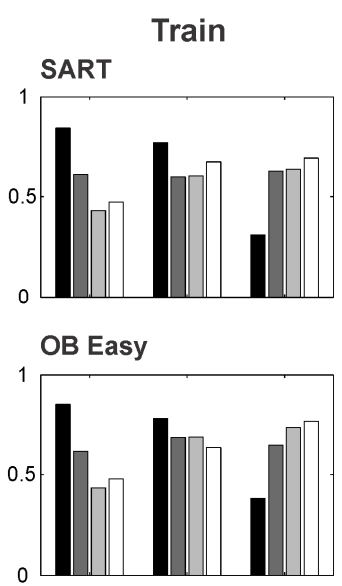

OB Hard

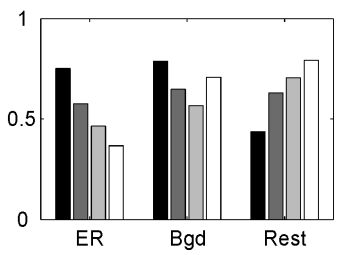

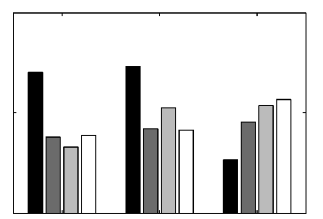

Test
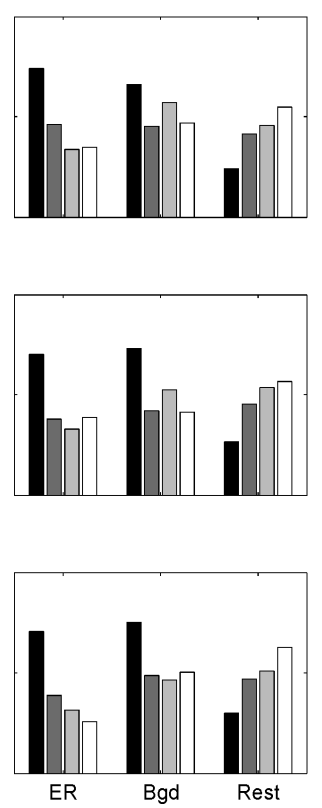

(b) Classification based on Phase features of the band-limited ICs
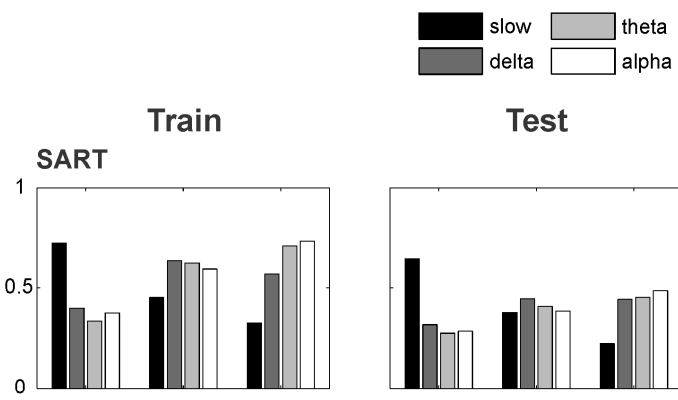

Test

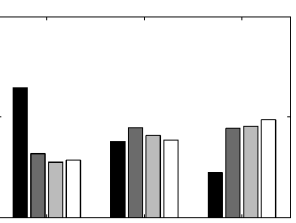

OB Easy

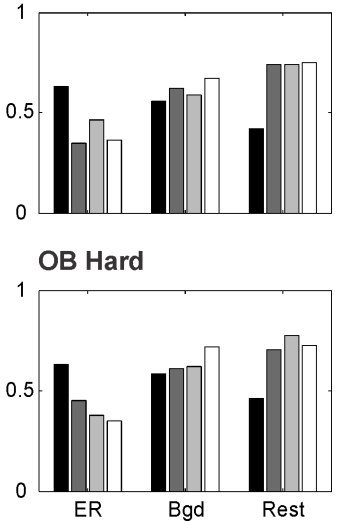

Fig. 7 - Sensitivity of classification for the training and test datasets, for the three task conditions based on: (a) the amplitude and (b) the phase features of the band-limited ICs. 
(a)
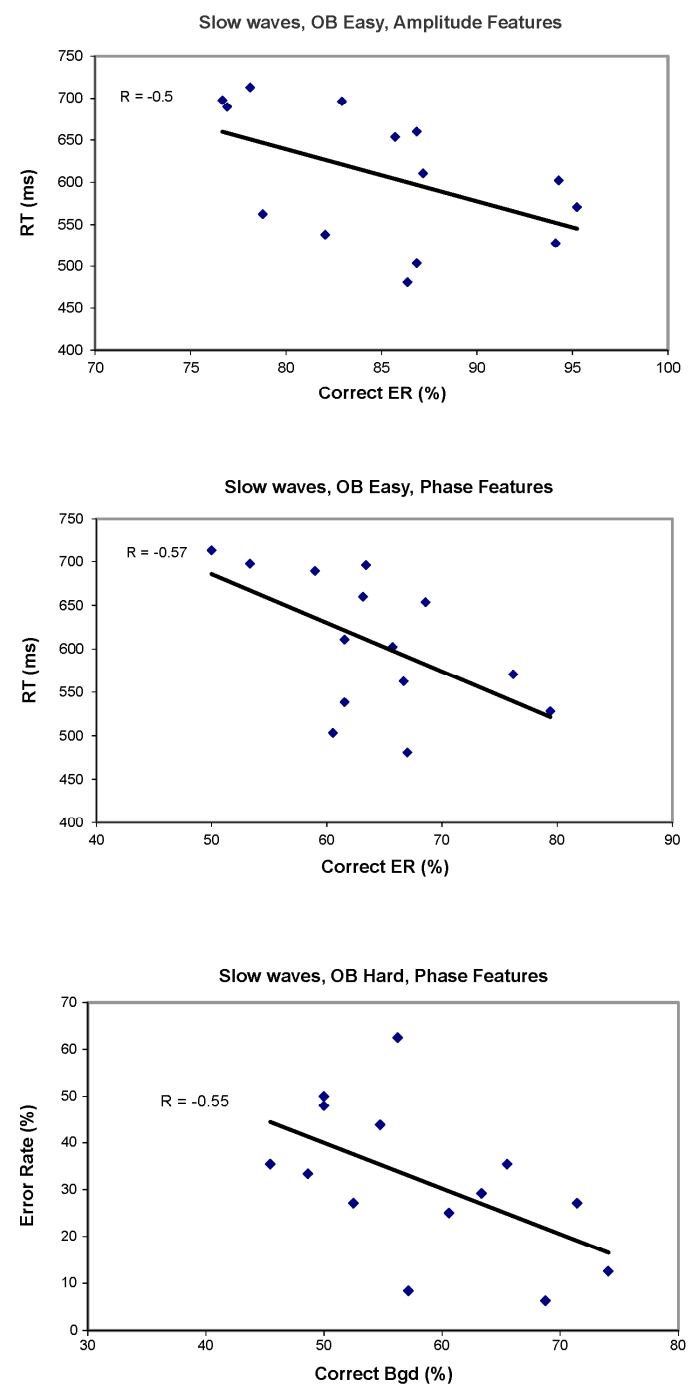

(b)
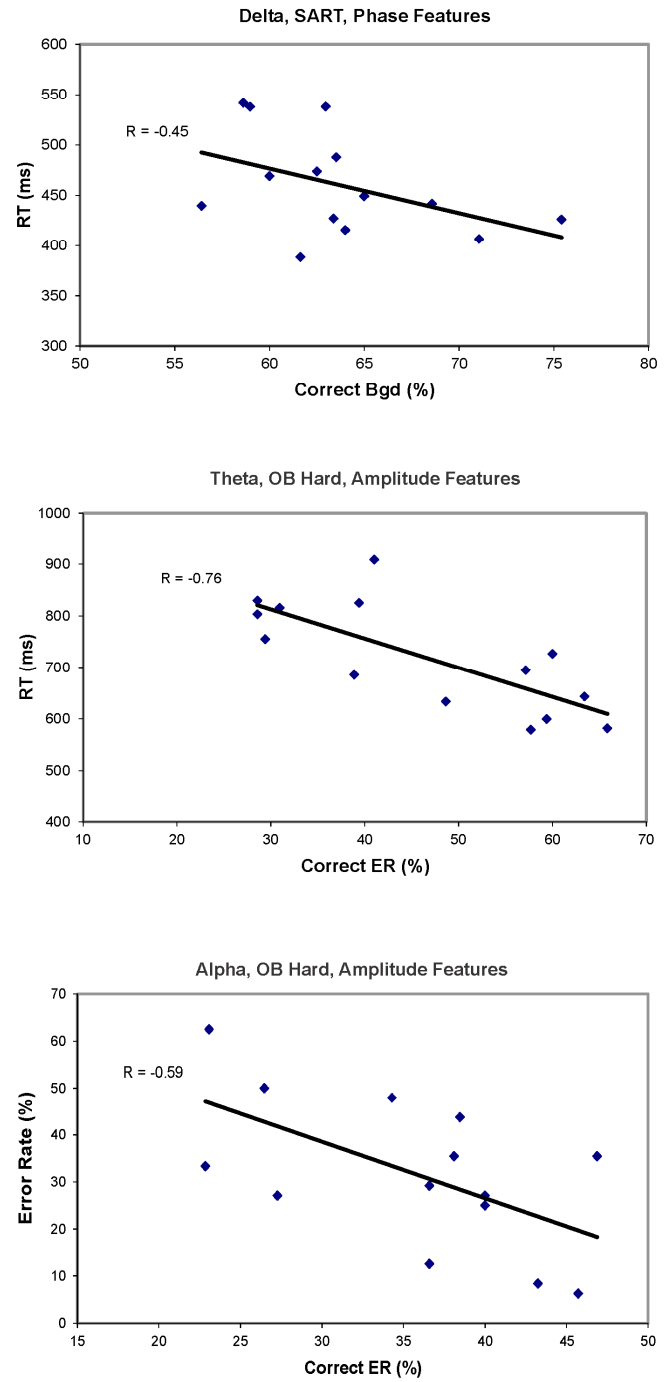

Fig. 8 - Sensitivity of classification based on (a) the slow wave amplitude or phase features, and (b) delta, theta and alpha bands amplitude or phase features, is negatively correlated with behavioural data $(p<0.05)$. 


\section{Appendix}

\section{The Itakura-Saito Distance}

For classification purposes, the distance between ICs (i.e. the degree of similarity between the ICs belonging to one class and dissimilarity between ICs of different classes) needed to be estimated. Note that the Euclidean distance between the auto regressive (AR) models is not an appropriate measure because the AR parameters of two separate time series may be heavily correlated and this could potentially lead to artificial similarity. A measure that quantifies the similarity between the signals being modelled, rather than the similarities between the pairs of model parameters alone, is required; for this reason the Itakura-Saito Distance was employed in this work (Itakura, 1975; Estrada et al., 2005).

\section{Neuroscale algorithm}

We made use of the supervised version of neuroscale algorithm developed by Low and Tipping (1996:1997), since this allows for the inclusion of subjective knowledge in the training process, which could, at varying degrees, affect the objective topology generated by the spatial input data. Here the distance being used for training $\delta_{i j}$ is defined as

$$
\delta_{i j}=(1-\alpha) d_{i j}^{*}+\alpha s_{i j},
$$

$d_{i j}^{*}$ is the Itakura-Saito distance matrix and $s_{i j}$ is another distance metric, where the distance between points in the same class is set to zero, while that between points in different classes is non-zero (Nabney, 2004). Our study entails three main classes. Based on prior knowledge regarding the ICs morphology and type of brain activity in the three conditions, the distance between ER and Background was assigned to one (since these two classes, though different, both represent task activity), distance between Background and Rest was 2 whereas that between ER and Rest was set to 3 (since brain activity during rest was expected to differ more from event-related than from background activity during task), as shown in Figure 2 (d). Note that these numbers for matrix $s$ have been chosen to indicate expected relative differences between the three classes. The parameter $\alpha$ varies from $0-$ the original, objective, unsupervised method based solely on the distribution of the original data, to $1-$ the completely supervised method that is no longer explicitly dependent on the data distribution. A value of $\alpha=0.5$ was chosen since it struck a balance between objective and subjective approaches, hence maintaining the spatial topology of the original data whilst improving the visualisation of the feature (Lowe and Tipping, 1996).

\section{Gaussian Mixture Models}

A set of GMMs (Nabney, 2004) were obtained in order to define the distributions of the 2-D neuroscale output, which indicated that the task ER and Background classes were separable whereas the Rest had some overlap with both. Therefore, the assumption used here was that the task ER and Background data could be confidently labelled as process A and B respectively, whereas from the Rest data there could be unlabelled points that belonged to either labelled sets. Therefore, the structure was first set up and the two GMMs of the labelled data were trained. Then these GMMs, derived from the two definite (strong) processes, were used to train a new GMM for the unlabelled data.

\section{Datasets for the Overall Results}

For the Go No-go task, 5 minute data segments were considered, namely the first 5 minutes, the second 5 minutes and an overlap (2.5-7.5 minutes) for each task block. Since the protocol remained the same throughout the task, the ICs extracted from the two task blocks were grouped together. For 
the rest condition, ICs were extracted from a 5 minute data segment $(0.5-5.5$ minutes into the rest block). This was carried out for every participant.

For the oddball task, TDSEP and hierarchical clustering were applied to three 4-minute segments $\left(1^{\text {st }} 4\right.$ minutes, $2^{\text {nd }} 4$ minutes and an overlap - i.e. 2 to 6 minutes) consecutively, for the Easy and the Hard task blocks separately, and for every participant. The Rest ICs were extracted from a 4-minute segment (1-5 minutes into the rest block). Note that both the Easy and Hard task environments shared the same rest period, hence their databanks contained the same Rest ICs.

For each task, the ICs of 14 participants were grouped together, forming the training databank. The ICs of the remaining 6 participants formed the test databank. 


\section{Supplementary Material}

Supplementary Table 1 - Median and range of RT, \% Error Rate and \% False Alarms (w.r.t. standard stimuli) across participants for the OB Easy and Hard task conditions. Wilcoxon signed-rank test statistics show statistically significant behavioural measures for the two conditions.

\begin{tabular}{|c|c|c|c|c|}
\hline & & Oddball Easy & Oddball Hard & Easy vs Hard \\
\hline \multirow[t]{2}{*}{ RT(ms) } & Median & 581.21 & 675.27 & $p<0.0001$ \\
\hline & Range & {$\left[\begin{array}{ll}480.75 & 712.87\end{array}\right]$} & {$\left[\begin{array}{ll}571.69 & 908.44\end{array}\right]$} & $z=-3.92$ \\
\hline \multirow{2}{*}{$\begin{array}{l}\text { Error Rate } \\
(\%)\end{array}$} & Median & 0 & 31.25 & $p<0.0001$ \\
\hline & Range & {$\left[\begin{array}{ll}0 & 16.67\end{array}\right]$} & {$\left[\begin{array}{ll}6.25 & 66.67\end{array}\right]$} & $z=-3.92$ \\
\hline \multirow{2}{*}{$\begin{array}{l}\text { False Alarms } \\
(\%)\end{array}$} & Median & 0 & 1.48 & $p<0.0001$ \\
\hline & Range & {$\left[\begin{array}{ll}0 & 1.97\end{array}\right]$} & {$\left[\begin{array}{ll}0 & 10.2\end{array}\right]$} & $z=-3.52$ \\
\hline
\end{tabular}




\section{Supplementary figures}

(a)

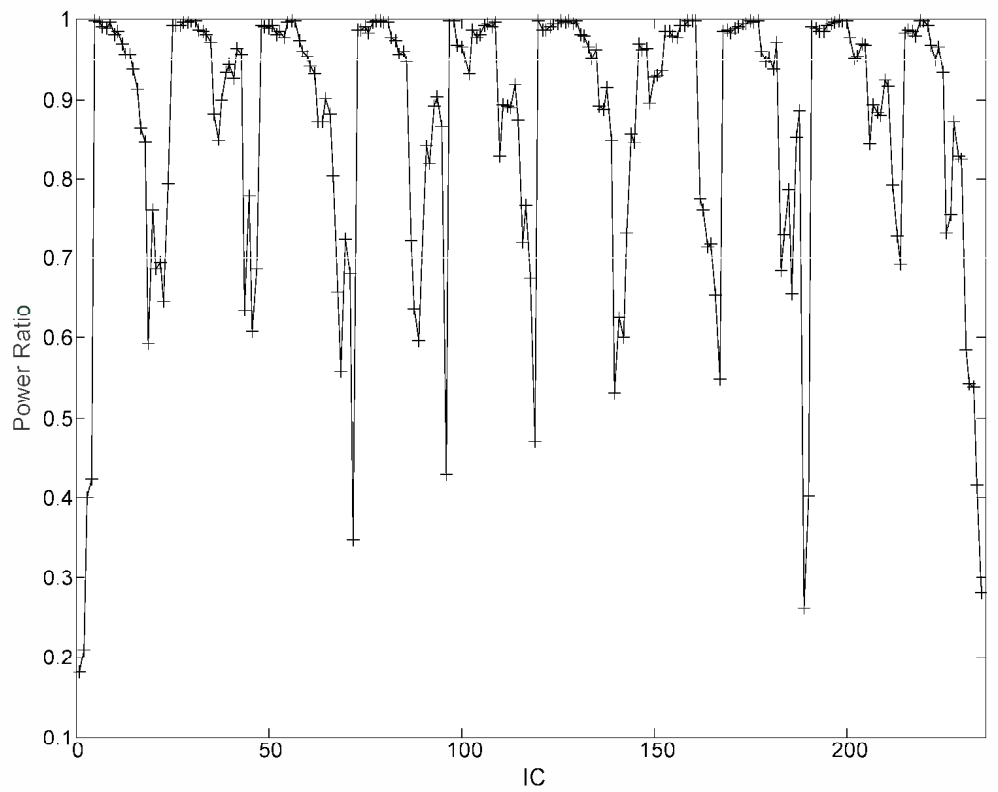

(b)
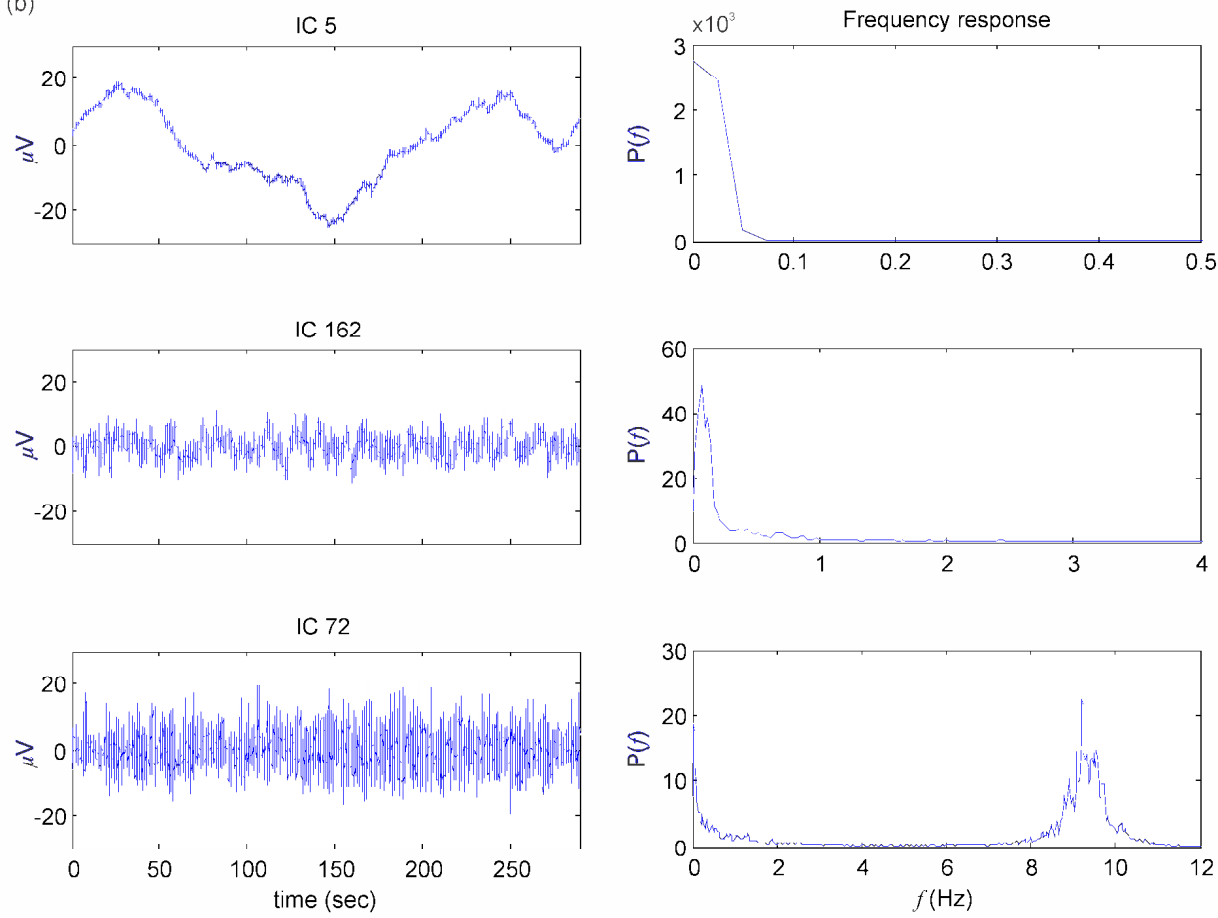

Fig. S1 - Ordering of Rest ICs based on their frequency content: (a) When the ratio of power in the $(0<f<1 \mathrm{~Hz})$ band to the power from $0-f_{s} / 2$ was greater than 0.95 , the ICs were marked as slow ICs. For power ratios between 0.7 and 0.95 the ICs had an intermediate frequency content. When the power ratio was less than 0.7 the ICs had a predominantly high (alpha) frequency content; (b) An example of each type of IC and their power spectral density. 

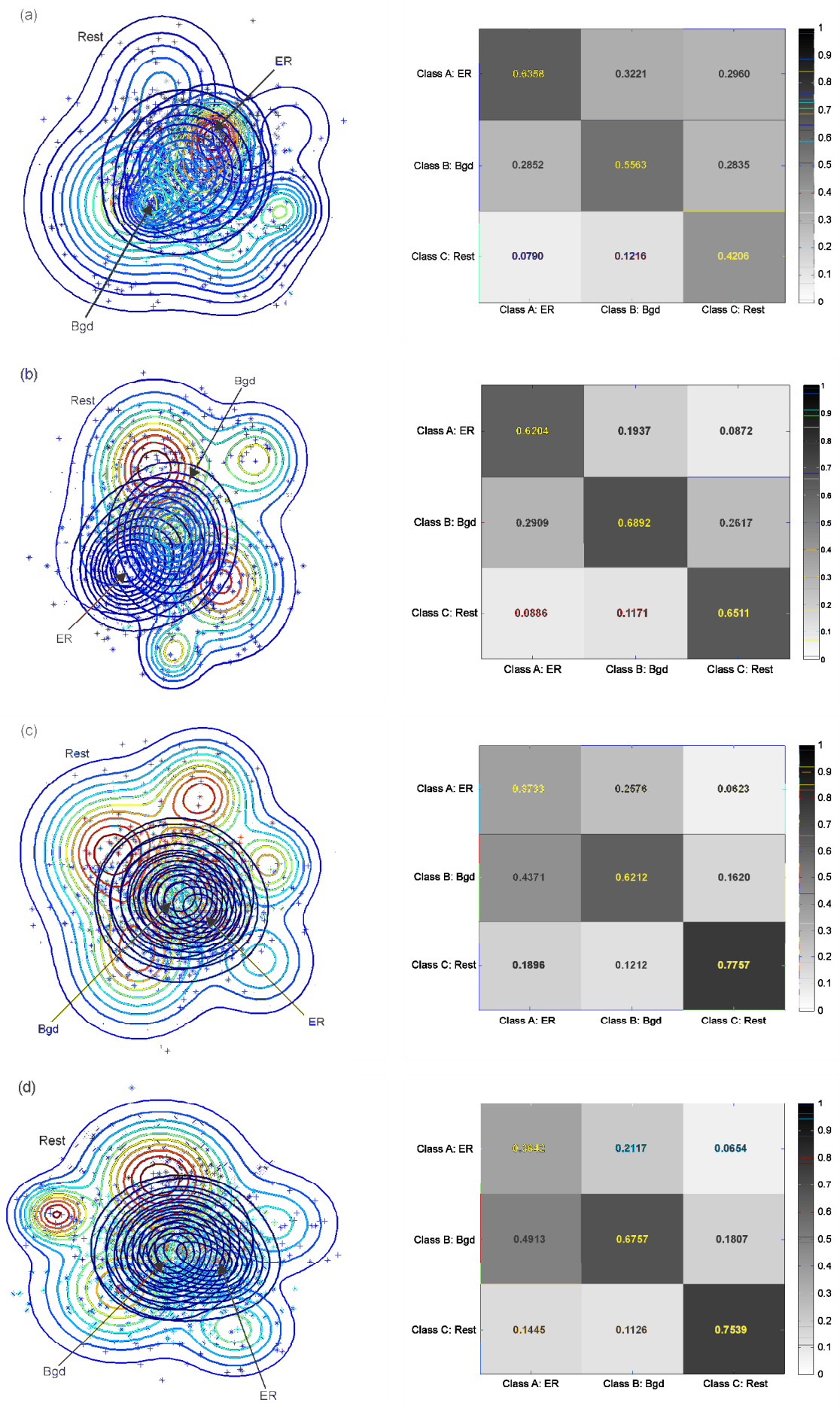

Fig. S2 - Training data classification results based on (a) Slow Wave Phase features of the ICs for the Oddball Easy task environment; (b) Delta f-Band Amplitude features of the ICs for the Oddball Easy task environment; (c) Theta $f$ Band Phase features of the ICs for the Oddball Hard task environment; (d) Alpha f-Band Phase features of the ICs for the Oddball Easy task environment. $1^{\text {st }}$ column: GMM contour plots for the Neuroscale output. $2^{\text {nd }}$ column: Confusion map showing sensitivity of classification on the diagonal (derived from the GMMs posterior probabilities for the three classes as explained in the main text). 
(a)
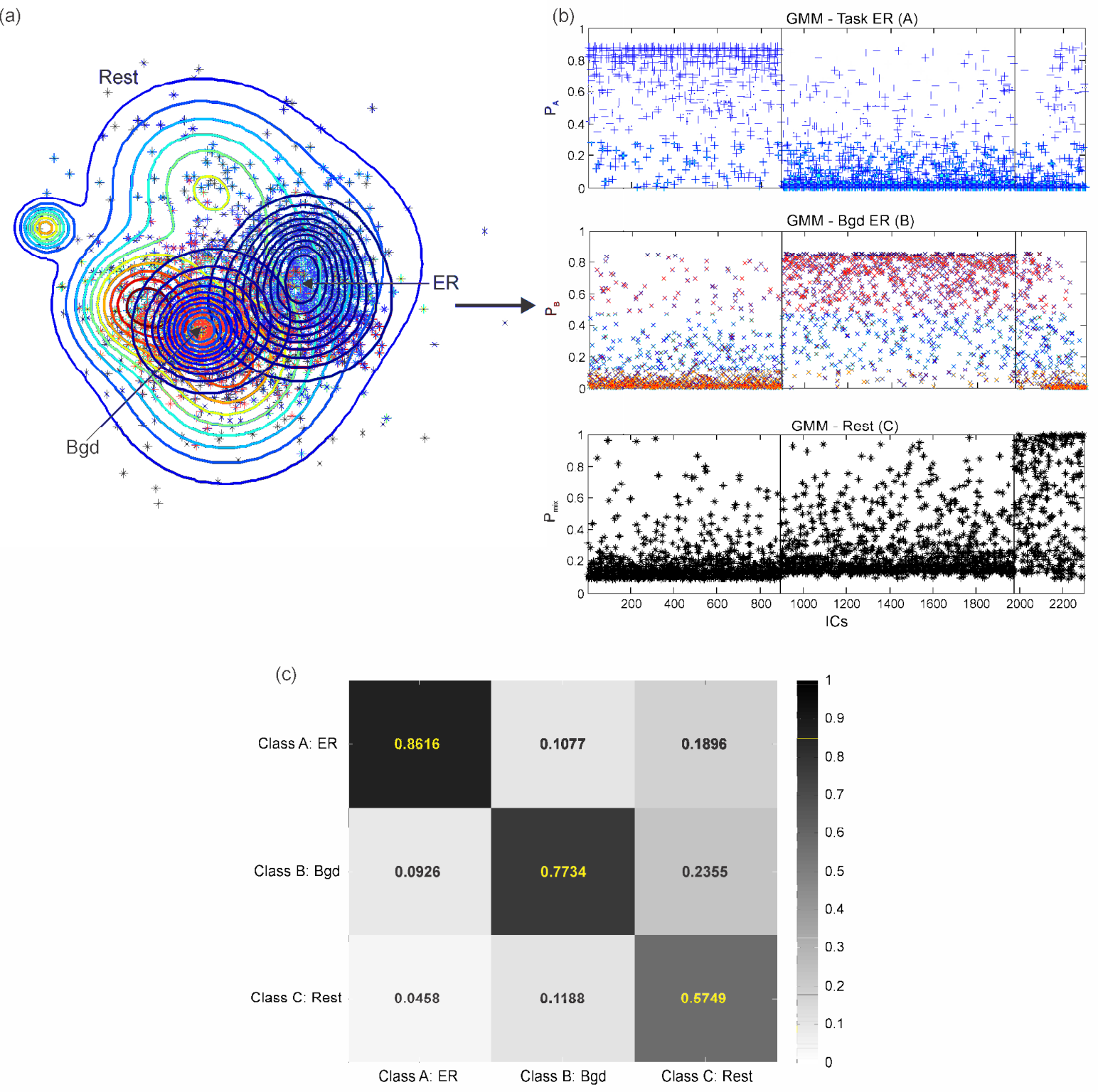

Fig. S3 - Training data classification results based on All f-Bands Amplitude features of the ICs for the SART task environment: (a) GMM contour plots for the Neuroscale output, (b) Posterior probabilities from the GMMs of the three classes, (c) Confusion map showing sensitivity of classification on the diagonal. 